\title{
Three-dimensional lattice multiflavor scalar chromodynamics: interplay between global and gauge symmetries
}

\author{
Claudio Bonati, ${ }^{1}$ Andrea Pelissetto, ${ }^{2}$ and Ettore Vicari ${ }^{1}$ \\ ${ }^{1}$ Dipartimento di Fisica dell'Università di Pisa and INFN Largo Pontecorvo 3, I-56127 Pisa, Italy \\ ${ }^{2}$ Dipartimento di Fisica dell'Università di Roma Sapienza and INFN Sezione di Roma I, I-00185 Roma, Italy
}

(Dated: January 7, 2020)

\begin{abstract}
We study the nature of the finite-temperature transition of the three-dimensional scalar chromodynamics with $N_{f}$ flavors. These models are constructed by considering maximally $\mathrm{O}(M)$-symmetric multicomponent scalar models, whose symmetry is partially gauged to obtain $\mathrm{SU}\left(N_{c}\right)$ gauge theories, with a residual nonabelian global symmetry given by $\mathrm{U}\left(N_{f}\right)$ for $N_{c} \geq 3$ and $\operatorname{Sp}\left(N_{f}\right)$ for $N_{c}=2$, so that $M=2 N_{c} N_{f}$. We find that their finite-temperature transition is continuous for $N_{f}=2$ and for all values of $N_{c}$ we investigated, $N_{c}=2,3,4$. Such continuous transitions belong to universality classes related to the global symmetry group of the theory. For $N_{c}=2$ it belongs to the $\mathrm{SO}(5)=$ $\mathrm{Sp}(2) / \mathbb{Z}_{2}$ universality class, while for $N_{c} \geq 3$ it belongs to the $\mathrm{SO}(3)=\mathrm{SU}(2) / \mathbb{Z}_{2}$ universality class. For $N_{f} \geq 3$, the transition is always of first order. These results match the predictions obtained by using the effective Landau-Ginzburg-Wilson approach in terms of a gauge-invariant order parameter. Our results indicate that the nonabelian gauge degrees of freedom are irrelevant at the transition. These conclusions are supported by an analysis of gauge-field dependent correlation functions, that are always short-ranged, even at the transition.
\end{abstract}

\section{INTRODUCTION}

The importance of symmetries in modern physics can be hardly overestimated. Global symmetries and the way in which they are realized are commonly used to identify and describe different phases of matter [1]. Local gauge symmetries play a fundamental role both in particle physics, where they lie at the heart of the Standard Model [2], and in condensed-matter physics, where their applications span from superconductivity 3] to topological order and quantum phase transitions [4].

Several systems of physical interest display both global and local symmetries, and a fundamental problem is to understand which of these symmetries play a role in determining the universal behavior of the system close to a continuous phase transition. The traditional LandauGinzburg-Wilson (LGW) approach to critical phenomena relies on statistical field theory [5-9]. In this scheme critical properties depend only on the global symmetry breaking pattern and on some "kinematic" parameters, like the space dimensionality and the number of fields components. For transitions to/from topologically ordered states this time-honored scheme has to be modified, due to the peculiar nonlocal character of topological order [10, 11]. However, when a continuous phase transition emerges due to the breaking of a global symmetry in a gauge theory, it is by no means obvious which is the role played by the gauge degrees of freedom: do they affect the critical behavior or not?

The study of the chiral phase transition in massless Quantum Chromodynamics (QCD) was likely the first occasion in which this problem could have been raised. Massless QCD is indeed invariant under local $\mathrm{SU}(3)$ color transformations and under global $\mathrm{SU}_{L}\left(N_{f}\right) \times$ $\mathrm{SU}_{R}\left(N_{f}\right)$ flavor transformations, with the chiral transition being associated with the symmetry breaking pat- tern $\mathrm{SU}_{L}\left(N_{f}\right) \times \mathrm{SU}_{R}\left(N_{f}\right) \rightarrow \mathrm{SU}_{V}\left(N_{f}\right)$ [2]. However, starting from the seminal work of Pisarski and Wilczek [12] (see Refs. [13, 14] for some refinements), it was always implicitly assumed that gauge degrees of freedom are irrelevant at the chiral transition, whose properties were predicted by using a gauge-invariant order parameter and the LGW approach. Numerical lattice results later supported these predictions, although with limited numerical precision because of the computational burden of simulating dynamical fermions. Moreover, in recent times possible hints of discrepancies have appeared (see Refs. [15, 16] for recent reviews).

The dependence of the critical behavior on the gauge degrees of freedom can be numerically investigated much more accurately in scalar models. The three dimensional (3D) abelian case attracted much attention in the recent past, both from the theoretical and from the numerical point of view [10, 11, 17 -25]. In particular, some works 22 25] reported some numerical evidence that the LGW approach, based on a gauge invariant order parameter, may not describe the emerging critical behavior.

Notwithstanding their applications to high-energy physics (most notably to the QCD chiral phase transition but also to possible extensions of the standard model) and their growing importance in condensedmatter physics [4, 11, 26, 27], 3D nonabelian gauge theories has been so far much less studied. The only case that was systematically investigated was that of the $3 \mathrm{D} \mathrm{SU}(2)$ gauge theory coupled to a scalar $\mathrm{SU}(2)$ doublet, which is relevant for the electroweak phase transition (see, e.g., Refs. 28 32]). For our purposes, however, this model is somehow trivial, since it is known that its phase diagram consists of a single phase [33 35].

To improve on this state of affairs, in Ref. [36] we presented results regarding a multiflavor 3D lattice scalar model with continuous $\mathrm{U}\left(N_{f}\right)$ symmetry, which might be called lattice multiflavor scalar chromodynamics. We de- 
termined the transitions in this model, investigated their nature, and compared the results with the predictions of two field-theoretical formalisms, the gauge-invariant LGW scheme and the continuum scalar chromodynamics. The outcome of this analysis was that the LGW approach correctly predicts the finite-temperature critical behavior of 3D multiflavor scalar chromodynamics in all cases we studied, i.e., for $N_{c}=2,3,4$ and $N_{f}=2,3$. The analysis of the lattice results reported in Ref. 36] was however necessarily sketchy, and in this paper we report all the analyses that permitted us to unambiguously identify the order of the transitions and the universality class in the case of continuous transitions. A more detailed discussion of the symmetries of the model, and in particular of the U(1) flavor symmetry, is also reported, together with the full details of the LGW approach for $N_{c}=2$, in which case the global symmetry of the model is $\operatorname{Sp}\left(N_{f}\right)$.

The paper is organized as follows. In Sec. IIIthe lattice multiflavor scalar chromodynamics model is introduced, with a discussion of its global and local symmetries. In Sec. III we discuss the predictions of the effective LGW approach. In Sec. IV we describe the lattice observables adopted and we briefly summarize the finite-size scaling (FSS) results we use in the analysis of the data. In Sec. $\nabla$ we present the results of the numerical simulations, and finally we draw our conclusions in Sec. VI. In App. @ we discuss the symplectic order parameters and, for $N_{f}=2$, the relation between $\mathrm{Sp}(2)$ and $\mathrm{O}(5)$ observables. App. B is devoted to a discussion of the LGW approach for the two-color case in which the global symmetry group is $\operatorname{Sp}\left(N_{f}\right)$. Finally, in App. C] we discuss some properties of the model for $\beta \rightarrow \infty$.

\section{THE LATTICE MODEL}

The three-dimensional lattice model we are going to study has $N_{c} \times N_{f}$ complex matrix variables $Z_{\boldsymbol{x}}^{a f}$ associated with each site $\boldsymbol{x}$ of a cubic lattice. Our starting point is the lattice model defined by the action

$$
\begin{aligned}
& S_{\mathrm{inv}}=-J \sum_{\boldsymbol{x}, \mu} \operatorname{Re} \operatorname{Tr} Z_{\boldsymbol{x}}^{\dagger} Z_{\boldsymbol{x}+\hat{\mu}}+\sum_{\boldsymbol{x}} V\left(\operatorname{Tr} Z_{\boldsymbol{x}}^{\dagger} Z_{\boldsymbol{x}}\right), \\
& V(X)=r X+u X^{2} .
\end{aligned}
$$

In Eq. (1) the first sum is over the lattice links, the second one is over the lattice sites, and $\hat{\mu}=\hat{1}, \hat{2}, \hat{3}$ are unit vectors along the three lattice directions. In particular, we consider the unit-length limit of the site variables, which is formally obtained by setting $r=-u$, and taking the limit $u \rightarrow \infty$ in the potential (2), so that the variables $Z$ satisfies

$$
\operatorname{Tr} Z_{\boldsymbol{x}}^{\dagger} Z_{\boldsymbol{x}}=1
$$

and the action simplifies to

$$
S_{\mathrm{inv}}=-J \sum_{\boldsymbol{x}, \mu} \operatorname{Re} \operatorname{Tr} Z_{\boldsymbol{x}}^{\dagger} Z_{\boldsymbol{x}+\hat{\mu}}
$$

Models with actions (11) and (4) are invariant under $\mathrm{O}(M)$ transformations with $M=2 N_{c} N_{f}$. This is immediately checked if we write the matrices $Z_{\boldsymbol{x}}$ in terms of $M$-component real vectors $\boldsymbol{S}_{\boldsymbol{x}}$. In the new variables, we obtain the standard $\mathrm{O}(M)$ nonlinear $\sigma$-model

$$
S_{M}=-J \sum_{\boldsymbol{x}, \mu} \boldsymbol{S}_{\boldsymbol{x}} \cdot \boldsymbol{S}_{\boldsymbol{x}+\hat{\mu}}, \quad \boldsymbol{S}_{\boldsymbol{x}} \cdot \boldsymbol{S}_{\boldsymbol{x}}=1 .
$$

We now proceed by gauging some of the degrees of freedom. We associate an $\mathrm{SU}\left(N_{c}\right)$ matrix $U_{\boldsymbol{x}, \hat{\mu}}$ with each lattice link and extend the action (4) to ensure $\mathrm{SU}\left(N_{c}\right)$ gauge invariance. We also add a kinetic term for the gauge variables in the Wilson form [37]. We obtain the model with action

$$
\begin{aligned}
S_{g} & =-\beta N_{f} \sum_{\boldsymbol{x}, \mu} \operatorname{Re} \operatorname{Tr}\left[Z_{\boldsymbol{x}}^{\dagger} U_{\boldsymbol{x}, \hat{\mu}} Z_{\boldsymbol{x}+\hat{\mu}}\right] \\
& -\frac{\beta_{g}}{N_{c}} \sum_{\boldsymbol{x}, \mu>\nu} \operatorname{Re} \operatorname{Tr}\left[U_{\boldsymbol{x}, \hat{\mu}} U_{\boldsymbol{x}+\hat{\mu}, \hat{\nu}} U_{\boldsymbol{x}+\hat{\nu}, \hat{\mu}}^{\dagger} U_{\boldsymbol{x}, \hat{\nu}}^{\dagger}\right],
\end{aligned}
$$

and partition function

$$
Z=\sum_{\{Z, U\}} e^{-S_{g}} .
$$

Note that the gauge group is $\mathrm{SU}\left(N_{c}\right)$ and not $\mathrm{U}\left(N_{c}\right)$, so that, for $N_{c}=1$, the model is not related to the $3 \mathrm{D}$ $\mathrm{CP}^{N_{f}-1}$ model 9] or to the abelian Higgs model, studied, e.g., in Ref. [21]. The factor $N_{f}$ in the first term is introduced so that the large- $N_{f}$ limit can be performed by keeping $\beta$ fixed; the factor $1 / N_{c}$ in the second term is instead conventional in the lattice gauge theory literature. Note that, for $\beta_{g} \rightarrow \infty$, the product of the gauge fields along a plaquette converges to one, and therefore we can set $U_{\boldsymbol{x}, \hat{\mu}}=1$ modulo a gauge transformation. Therefore, in this limit we reobtain the $\mathrm{O}(M)$ invariant theory (4) we started from.

It is immediate to see that, for any value of $N_{c}$ and $N_{f}, S_{g}$ is invariant under the local gauge transformation

$$
Z_{\boldsymbol{x}} \rightarrow G_{\boldsymbol{x}} Z_{\boldsymbol{x}}, \quad U_{\boldsymbol{x}, \hat{\mu}} \rightarrow G_{\boldsymbol{x}} U_{\boldsymbol{x}, \hat{\mu}} G_{\boldsymbol{x}+\hat{\mu}}^{\dagger},
$$

with $G_{\boldsymbol{x}} \in \mathrm{SU}\left(N_{c}\right)$, and under the global transformation

$$
Z_{\boldsymbol{x}} \rightarrow Z_{\boldsymbol{x}} V, \quad U_{\boldsymbol{x}, \hat{\mu}} \rightarrow U_{\boldsymbol{x}, \hat{\mu}}
$$

with $V \in \mathrm{U}\left(N_{f}\right)$. Note that, more precisely, the global symmetry group of the model is $\mathrm{U}\left(N_{f}\right) / \mathbb{Z}_{N_{c}}$, where $\mathbb{Z}_{N_{c}}$ is the center of the gauge symmetry group $\mathrm{SU}\left(N_{c}\right)$.

Actually, for $N_{c}=2$ the action $S_{g}$ is invariant under a larger global symmetry group, the compact complex symplectic group $^{1} \mathrm{Sp}\left(N_{f}\right)$. This is a well established result (we found mention of it, in various forms, e.g., in

\footnotetext{
${ }^{1}$ Several notations are used to denote this group: in particular both $\operatorname{Sp}\left(N_{f}\right)$ and $\operatorname{Sp}\left(2 N_{f}\right)$ can be found in the literature.
} 
Refs. [11, 38 40]), which is a consequence of the selfduality of the fundamental representation of $\mathrm{SU}(2)$. We will here briefly explain the origin of this symmetry enlargement, introducing also some notations that will be useful in the following.

We define

$$
Y_{\boldsymbol{x}}^{a f}=\sum_{b} \epsilon^{a b} \bar{Z}_{\boldsymbol{x}}^{b f}
$$

where $\epsilon^{a b}$ is the completely antisymmetric tensor in 2 dimensions $\left(\epsilon^{12}=-\epsilon^{21}=1\right)$, and the $2 \times 2 N_{f}$ matrix field $\Gamma_{\boldsymbol{x}}^{a \alpha}$, defined by

$$
\Gamma_{\boldsymbol{x}}^{a \alpha}= \begin{cases}Z_{\boldsymbol{x}}^{a \alpha} & \text { if } 1 \leq \alpha \leq N_{f} \\ Y_{\boldsymbol{x}}^{a\left(\alpha-N_{f}\right)} & \text { if } N_{f}+1 \leq \alpha \leq 2 N_{f}\end{cases}
$$

Since SU(2) matrices satisfy

$$
\sum_{b} \epsilon^{a b} \bar{U}^{b c}=\sum_{b} U^{a b} \epsilon^{b c}
$$

$\Gamma_{\boldsymbol{x}}$ transforms covariantly under gauge transformations:

$$
\Gamma_{\boldsymbol{x}} \rightarrow G_{\boldsymbol{x}} \Gamma_{\boldsymbol{x}}
$$

We can now rewrite the nearest-neighbor interaction term involving the scalar variables as

$$
\begin{aligned}
& \frac{1}{2} \sum_{f, a, b}\left[\bar{Z}_{\boldsymbol{x}}^{a f} U_{\boldsymbol{x}, \hat{\mu}}^{a b} Z_{\boldsymbol{x}+\hat{\mu}}^{b f}+Z_{\boldsymbol{x}}^{a f} \bar{U}_{\boldsymbol{x}, \hat{\mu}}^{a b} \bar{Z}_{\boldsymbol{x}+\hat{\mu}}^{b f}\right]= \\
& \frac{1}{2} \sum_{f, a, b}\left[\bar{Z}_{\boldsymbol{x}}^{a f} U_{\boldsymbol{x}, \hat{\mu}}^{a b} Z_{\boldsymbol{x}+\hat{\mu}}^{b f}+\bar{Y}_{\boldsymbol{x}}^{a f} U_{\boldsymbol{x}, \hat{\mu}}^{a b} Y_{\boldsymbol{x}+\hat{\mu}}^{b f}\right]= \\
& \frac{1}{2} \sum_{\gamma, a, b} \bar{\Gamma}_{\boldsymbol{x}}^{a \gamma} U_{\boldsymbol{x}, \hat{\mu}}^{a b} \Gamma_{\boldsymbol{x}+\hat{\mu}}^{b \gamma}=\frac{1}{2} \operatorname{Tr} \Gamma_{\boldsymbol{x}}^{\dagger} U_{\boldsymbol{x}, \hat{\mu}} \Gamma_{\boldsymbol{x}+\hat{\mu}}
\end{aligned}
$$

Apparently, the action (14) is invariant under the global transformations

$$
\Gamma_{\boldsymbol{x}} \rightarrow \Gamma_{\boldsymbol{x}} M, \quad M \in \mathrm{U}\left(2 N_{f}\right)
$$

However, one should bear in mind that the $\Gamma$ variables are not generic, since they are obtained by a formal doubling of the degrees of freedom. Therefore, one must only consider transformations $M$ that maintain the particular structure (11). To identify them, we note that the previous bipartite structure of $\Gamma$ is equivalent to the relation

$$
\sum_{a} \epsilon^{a b} \bar{\Gamma}_{\boldsymbol{x}}^{b \alpha}=-\sum_{\gamma} \Gamma_{\boldsymbol{x}}^{a \gamma} J^{\gamma \alpha}
$$

where $J$ is the $2 N_{f} \times 2 N_{f}$ matrix

$$
J=\left(\begin{array}{cc}
0 & -I \\
I & 0
\end{array}\right)
$$

and $I$ is the $N_{f} \times N_{f}$ identity matrix. Therefore, the global invariance group of $S_{g}$ is the subgroup of $\mathrm{U}\left(2 N_{f}\right)$ which leaves invariant the relation Eq. (16). By straightforward manipulations it is possible to show that this requires $M$ to satisfy

$$
M J M^{T}=J,
$$

which identifies the global symmetry group as the compact (unitary) complex symplectic group $\operatorname{Sp}\left(N_{f}\right)$ (see, e.g., Ref. [41]). The global symmetry group for $N_{c}=2$ is thus $\operatorname{Sp}\left(N_{f}\right) / \mathbb{Z}_{2}$, since the sign of the field can be redefined by a gauge transformation. Note that, for $N_{f}=2$, we have the isomorphism (see, e.g., Ref. [41])

$$
\mathrm{SO}(5)=\operatorname{Sp}(2) / \mathbb{Z}_{2}
$$

Finally, let us explicitly note that the $\operatorname{Sp}\left(N_{f}\right)$ symmetry also holds when the fields do not satisfy the unit-length condition. Since

$$
\operatorname{Tr} Z_{\boldsymbol{x}}^{\dagger} Z_{\boldsymbol{x}}=\frac{1}{2} \operatorname{Tr} \Gamma_{\boldsymbol{x}}^{\dagger} \Gamma_{\boldsymbol{x}}
$$

is invariant under any $\mathrm{U}\left(2 N_{f}\right)$ transformations, and, in particular, under those of its $\operatorname{Sp}\left(N_{f}\right)$ subgroup, the action is $\operatorname{Sp}\left(N_{f}\right)$ invariant for generic site potentials $V$ in Eqs. (11) and (2).

\section{EFFECTIVE FIELD THEORY RESULTS}

The critical behavior of the lattice multiflavor scalar chromodynamics was discussed in Ref. [36]. Two different approaches were considered: the continuum theory corresponding to the lattice model and the LandauGinzburg-Wilson theory built in term of a gaugeinvariant order parameter. The renormalization-group flow of continuum multiflavor chromodynamics was studied in the $\varepsilon$-expansion around four dimensions [42]. It was found that a stable fixed point (FP) only exists for a very large number of flavors [for $N_{c}=2$ it exists only for $N_{f}>359+O(\varepsilon)$ ]. As a consequence, for small values of $N_{f}$ a first-order transition is predicted.

In the LGW approach, one starts by considering an order parameter that breaks the global symmetry of the model. We first consider the case $N_{c}>2$, so that the global symmetry is $\mathrm{U}\left(N_{f}\right) / \mathbb{Z}_{N_{c}}$. Since this is not a simple group, we may have different symmetry breakings.

One possibility is that of breaking the $\mathrm{SU}\left(N_{f}\right)$ subgroup. An appropriate order parameter is the field combination

$$
Q_{\boldsymbol{x}}^{f g}=\sum_{a} \bar{Z}_{\boldsymbol{x}}^{a f} Z_{\boldsymbol{x}}^{a g}-\frac{\delta^{f g}}{N_{f}}
$$

which is the natural generalization of the quantity studied in abelian models (see, e.g., Refs. [20, 21]). The corresponding LGW theory is obtained by considering a hermitian traceless $N_{f} \times N_{f}$ matrix field $\Psi(\boldsymbol{x})$, which represents a coarse-grained version of $Q_{\boldsymbol{x}}$, with Lagrangian

$$
\begin{aligned}
& \mathcal{L}_{\mathrm{LGW}}=\operatorname{Tr} \partial_{\mu} \Psi \partial_{\mu} \Psi+r \operatorname{Tr} \Psi^{2} \\
& \quad+u_{3} \operatorname{Tr} \Psi^{3}+u_{41} \operatorname{Tr} \Psi^{4}+u_{42}\left(\operatorname{Tr} \Psi^{2}\right)^{2} .
\end{aligned}
$$


This Lagrangian is invariant under the global transformations $\Psi \rightarrow V \Psi V^{\dagger}$ and therefore the symmetry group is $\mathrm{SU}\left(N_{f}\right) / \mathbb{Z}_{N_{f}}$. As discussed in, e.g., Ref. [20], the cubic term vanishes for $N_{f}=2$. In this case a continuous transition is possible in the $\mathrm{SU}(2) / \mathbb{Z}_{2}$, that is in the vector $\mathrm{SO}(3)$, universality class. For $N_{f}>2$ the cubic term is present and, on the basis of the usual mean-field arguments, one expects a first-order transition.

A second possibility is that of breaking the $\mathrm{U}(1) / \mathbb{Z}_{N_{c}}$ symmetry group associated with the transformations

$$
Z_{\boldsymbol{x}}^{a f} \rightarrow e^{i \alpha} Z_{\boldsymbol{x}}^{a f},
$$

which leave invariant the order parameter $Q_{x}^{a b}$. However, for $N_{f}<N_{c}$, this additional symmetry is only apparent. Indeed, for any $\boldsymbol{x}$, one can find an $\mathrm{SU}\left(N_{c}\right)$ matrix $G_{\boldsymbol{x}}$ such that

$$
e^{i \alpha} Z_{\boldsymbol{x}}=G_{\boldsymbol{x}} Z_{\boldsymbol{x}}
$$

If $N_{f}<N_{c}$, there is a gauge transformation $Z^{\prime}{ }_{\boldsymbol{x}}=$ $G_{1 x} Z_{\boldsymbol{x}}$ such that ${Z^{\prime}}_{\boldsymbol{x}}^{a f}=0$ for any $f$ and any $a$ satisfying $N_{f}+1 \leq a \leq N_{c}$. Then, one defines the $N_{c} \times N_{c}$ unitary matrix

$$
G_{2}=\operatorname{diag}\left(g_{1}, \ldots, g_{N_{c}}\right)
$$

with $g_{a}=e^{i \alpha}$ for $1 \leq a \leq N_{f}, g_{a}=e^{-i \alpha N_{f}}$ for $a=$ $N_{f}+1, g_{a}=1$ for $a>N_{f}+1$. It is then easy to verify that $G_{x}=G_{1 x}^{\dagger} G_{2} G_{1 x}$ satisfies Eq. (24).

For $N_{f} \geq N_{c}$, the relation (24) does not hold anymore, and one must consider the breaking of the abelian symmetry $\mathrm{U}(1) / \mathbb{Z}_{N_{c}}$. An appropriate order parameter is

$$
D_{\boldsymbol{x}}^{f_{1}, \ldots, f_{N_{c}}}=\sum_{a_{1}, \ldots, a_{N_{c}}} \epsilon^{a_{1}, \ldots, a_{N_{c}}} Z_{\boldsymbol{x}}^{a_{1} f_{1}} \ldots Z_{\boldsymbol{x}}^{a_{N_{c}} f_{N_{c}}}
$$

which is invariant under gauge transformations (here $\epsilon^{a_{1}, \ldots, a_{N_{c}}}$ is the completely antisymmetric tensor in $N_{c}$ dimensions). Such an order parameter vanishes for $N_{f}<$ $N_{c}$, an expected result given the effective absence of the symmetry in this case. For $N_{f}=N_{c}$ the order parameter defined in Eq. (26) is invariant under $\mathrm{SU}\left(N_{f}\right)$ transformations and therefore it is a good order parameter for the breaking of the $\mathrm{U}(1)$ flavor symmetry. It can be rewritten in a simpler way, as

$$
D_{\boldsymbol{x}}^{f_{1}, \ldots, f_{N_{c}}}=\epsilon^{f_{1}, \ldots, f_{N_{c}}} \operatorname{det} Z_{\boldsymbol{x}} .
$$

On the other hand, for $N_{f}>N_{c}$, the order parameter belongs to a nontrivial representation of $\mathrm{SU}\left(N_{f}\right)$. Therefore, it condenses only if both the $\mathrm{SU}\left(N_{f}\right)$ and the $\mathrm{U}(1)$ symmetries are broken.

As we discuss in App. C] in our model, for $N_{c} \geq 3$, the order parameter $D_{\boldsymbol{x}}^{f_{1}, \ldots, f_{N_{c}}}$ vanishes for $\beta \rightarrow \infty$. If we assume that the relevant configurations in the low-temperature phase are simply obtained by considering short-range fluctuations on top of the ordered background observed for $\beta=+\infty$, we conclude that
$D$-correlations are short-ranged in the low-temperature phase, i.e., that the $\mathrm{U}(1)$ symmetry is not broken. Below we will present numerical results for $N_{c}=N_{f}=3$ that confirm this picture.

For $N_{c}=2$ the symmetry group is $\operatorname{Sp}\left(N_{f}\right) / \mathbb{Z}_{2}$. The order parameter is a symplectic analogue of $Q_{\boldsymbol{x}}$. Specifically, we define

$$
\mathcal{T}_{\boldsymbol{x}}^{\alpha \beta}=\sum_{a} \bar{\Gamma}_{\boldsymbol{x}}^{a \alpha} \Gamma_{\boldsymbol{x}}^{a \beta}-\frac{\delta^{\alpha \beta}}{2 N_{f}} \sum_{a \gamma} \bar{\Gamma}_{\boldsymbol{x}}^{a \gamma} \Gamma_{\boldsymbol{x}}^{a \gamma},
$$

with $\Gamma_{\boldsymbol{x}}^{a \alpha}$ defined in Eq. (11). This order parameter is a $2 N_{f} \times 2 N_{f}$ hermitian traceless matrix which satisfies the additional condition

$$
J \overline{\mathcal{T}} J+\mathcal{T}=0,
$$

which follows from Eq. (16). The matrix $\mathcal{T}$ is thus an element of the $\mathfrak{s p}\left(N_{f}\right)$ algebra [41]. The explicit construction of the corresponding LGW theory starts by defining a $2 N_{f} \times 2 N_{f}$ hermitian traceless matrix field $\Psi(\boldsymbol{x})$ that satisfies the analog of Eq. (29). The corresponding LGW theory is obtained by considering the most general quartic polynomial in the fields: we reobtain Eq. (22). For $N_{f}=2$, as discussed in App. B the cubic term vanishes. Therefore, continuous transitions are allowed in the $\mathrm{SO}(5)$ universality class, given the isomorphism between $\mathrm{Sp}(2) / \mathbb{Z}_{2}$ and the $\mathrm{SO}(5)$ group. For $N_{f}>2$, a cubic operator is generically present and therefore we expect first-order transitions. Note, that for $N_{c}=2$, there is no residual $\mathrm{U}(1)$ symmetry, as $\mathrm{U}(1)$ global transformations are a subgroup of the $\operatorname{Sp}\left(N_{f}\right)$ group.

We finally note that the LGW approach based on the symmetry of the model does not depend on the specific form of the lattice potential $V(X)$ in Eq. (2). Moreover, we recall that the presence of a stable fixed point in the corresponding LGW theory does not exclude the possibility that the model undergoes a first-order transition, when the system is outside the attraction domain of the stable fixed point even though it shares the global symmetry of the universality class.

\section{OBSERVABLES AND ANALYSIS METHOD}

In this section we introduce the lattice observables studied and we briefly recall some basic facts about FSS that will be relevant for the analysis of the numerical data. We always assume the lattice to have periodic boundary conditions and to be of linear size $L$.

\section{A. Lattice observables}

In the following we consider the energy density and the specific heat, defined as

$$
E=\frac{1}{\beta N_{f} V}\left\langle S_{g}\right\rangle, \quad C=\frac{1}{\beta^{2} N_{f}^{2} V}\left(\left\langle S_{g}^{2}\right\rangle-\left\langle S_{g}\right\rangle^{2}\right),
$$


where $V=L^{3}$. We also define the average gauge energy as

$$
E_{g}=\frac{1}{6 V N_{c}}\left\langle\sum_{\boldsymbol{x}, \mu>\nu} \operatorname{Re} \operatorname{Tr}\left[U_{\boldsymbol{x}, \hat{\mu}} U_{\boldsymbol{x}+\hat{\mu}, \hat{\nu}} U_{\boldsymbol{x}+\hat{\nu}, \hat{\mu}}^{\dagger} U_{\boldsymbol{x}, \hat{\nu}}^{\dagger}\right]\right\rangle .
$$

To study the breaking of the $\mathrm{SU}\left(N_{f}\right)$ flavor symmetry we consider the order parameter $Q$ defined in Eq. (21), which is a hermitian and traceless $N_{f} \times N_{f}$ matrix. Its two-point correlation function is defined by

$$
G(\boldsymbol{x}-\boldsymbol{y})=\left\langle\operatorname{Tr} Q_{\boldsymbol{x}} Q_{\boldsymbol{y}}\right\rangle
$$

where the translation invariance of the system has been explicitly taken into account. We can define the correasponding susceptibility $\chi$ and correlation length $\xi$ as

$$
\begin{aligned}
\chi & =\sum_{\boldsymbol{x}} G(\boldsymbol{x}), \\
\xi^{2} & =\frac{1}{4 \sin ^{2}(\pi / L)} \frac{\widetilde{G}(\mathbf{0})-\widetilde{G}\left(\boldsymbol{p}_{m}\right)}{\widetilde{G}\left(\boldsymbol{p}_{m}\right)}
\end{aligned}
$$

where $\widetilde{G}(\boldsymbol{p})=\sum_{\boldsymbol{x}} e^{i \boldsymbol{p} \cdot \boldsymbol{x}} G(\boldsymbol{x})$ is the Fourier transform of $G(\boldsymbol{x})$ and $\boldsymbol{p}_{m}=(2 \pi / L, 0,0)$. We also consider the Binder parameter $U$, defined by

$$
U=\frac{\left\langle\mu_{2}^{2}\right\rangle}{\left\langle\mu_{2}\right\rangle^{2}}, \quad \mu_{2}=\frac{1}{V^{2}} \sum_{\boldsymbol{x}, \boldsymbol{y}} \operatorname{Tr} Q_{\boldsymbol{x}} Q_{\boldsymbol{y}}
$$

We will study the $\mathrm{U}(1)$ flavor symmetry only for $N_{f}=$ $N_{c}$. In this case it is equivalent to consider the scalar order parameter, see Eq. (27),

$$
D_{\boldsymbol{x}}=\operatorname{det} Z_{\boldsymbol{x}}
$$

We define the correlation function

$$
G_{D}(\boldsymbol{x}-y)=\left\langle\operatorname{Re} \bar{D}_{\boldsymbol{x}} D_{\boldsymbol{y}}\right\rangle
$$

the correlation length $\xi_{D}$ using the analogue of Eq. (34), and the Binder parameter

$$
U_{D}=\frac{\left\langle\mu_{D 2}^{2}\right\rangle}{\left\langle\mu_{D 2}\right\rangle^{2}}, \quad \mu_{D 2}=\frac{1}{V^{2}} \sum_{\boldsymbol{x}, \boldsymbol{y}} \operatorname{Re} \bar{D}_{\boldsymbol{x}} D_{\boldsymbol{y}}
$$

To better appreciate the role of the gauge degrees of freedom, we also study some observables involving the $\mathrm{SU}\left(N_{c}\right)$ gauge link variables. More specifically, we consider the averages

$$
\left\langle\sum_{a b} \bar{Z}_{\boldsymbol{x}}^{a f}\left[\prod_{\ell \in \mathcal{C}} U_{\ell}\right]^{a b} Z_{\boldsymbol{y}}^{b g}\right\rangle
$$

where the product extends over the link variables that belong to a lattice path $\mathcal{C}$ connecting the points $\boldsymbol{x}$ and $\boldsymbol{y}$. To define quantities that have the correct FSS, the path $\mathcal{C}$ must be chosen appropriately [43], and here we consider correlations between points along lattice lines:

$G_{V}(t, L)=\operatorname{Re}\left\langle\sum_{a b f g} \bar{Z}_{\boldsymbol{x}}^{a f}\left[\prod_{k=0}^{t-1} U_{\boldsymbol{x}+k \hat{\mu}, \hat{\mu}}\right]^{a b} Z_{\boldsymbol{x}+t \hat{\mu}}^{b f}\right\rangle$.

As usual, translation invariance and independence of the direction $\hat{\mu}$ can be used to actually increase the statistics. In some test cases we also determined the Polyakov loop

$$
P(L)=\frac{1}{3 L^{3}} \sum_{\boldsymbol{x}, \mu} \operatorname{Re}\left\langle\operatorname{Tr}\left[\prod_{k=0}^{L-1} U_{\boldsymbol{x}+k \hat{\mu}, \hat{\mu}}\right]\right\rangle .
$$

For $N_{f}=2$ and $N_{c}=2$ the model is invariant under $\operatorname{Sp}(2) / \mathbb{Z}_{2}=O(5)$ transformations. We discuss in App. A the $\mathrm{O}(5)$ observables that can be defined in terms of the order parameter (28). In particular, we show that the second-moment correlation length computed from $G(\boldsymbol{x})$, $G_{D}(\boldsymbol{x})$ or the $\mathrm{O}(5)$-invariant correlation frunction of the the order parameter $\mathcal{T}^{\alpha \beta}$ are numerically the same. For the Binder parameters, instead, the relation is not trivial. We have

$$
U=\frac{25}{21} U_{\Gamma}, \quad U_{D}=\frac{10}{7} U_{\Gamma}
$$

where $U_{\Gamma}$ is the $\mathrm{O}(5)$-invariant Binder parameter defined in App. A, which corresponds to the usual vector parameter in the $\mathrm{O}(5)$ theory.

For $N_{f}=2$ and $N_{c} \geq 3$, the global symmetry group is $\mathrm{SU}(2) / \mathbb{Z}_{2}=S O(3)$. This invariance can be more easily understood by defining the gauge-invariant threecomponent real vector variables $\varphi_{\boldsymbol{x}}^{k}$ as

$$
\varphi_{\boldsymbol{x}}^{k}=\sum_{a, f, g} \bar{Z}_{\boldsymbol{x}}^{a f} \sigma_{f g}^{k} Z_{\boldsymbol{x}}^{a g}=\sum_{f, g} \sigma_{f g}^{k} Q_{\boldsymbol{x}}^{f g}
$$

where $\sigma^{k}$ are the Pauli matrices. Previoulsy defined observables, like $\chi$ and $U$, can be rewritten in term of the vector variable $\boldsymbol{\varphi}_{\boldsymbol{x}}$ using

$$
\begin{aligned}
& G(\boldsymbol{x}-\boldsymbol{y})=\frac{1}{2}\left\langle\boldsymbol{\varphi}_{\boldsymbol{x}} \cdot \boldsymbol{\varphi}_{\boldsymbol{y}}\right\rangle, \\
& U=\frac{\left\langle\mu_{2}^{2}\right\rangle}{\left\langle\mu_{2}\right\rangle^{2}}, \quad \mu_{2}=\frac{1}{V^{2}} \sum_{\boldsymbol{x}, \boldsymbol{y}} \boldsymbol{\varphi}_{\boldsymbol{x}} \cdot \boldsymbol{\varphi}_{\boldsymbol{y}} .
\end{aligned}
$$

Note however that the vectors $\boldsymbol{\varphi}_{\boldsymbol{x}}$ do not have fixed length, indeed

$$
\boldsymbol{\varphi}_{\boldsymbol{x}} \cdot \boldsymbol{\varphi}_{\boldsymbol{x}}=2 \operatorname{Tr} Q_{\boldsymbol{x}}^{2} \leq 1
$$

\section{B. Finite-Size Scaling}

To investigate continuous transitions using FSS it is particularly convenient to study RG invariant quantities, such as $U$ and

$$
R_{\xi}=\xi / L
$$


For an RG-invariant quantity, generically denoted by $R$, FSS theory predicts the scaling behavior [8]

$$
\begin{aligned}
& R(\beta, L)=f_{R}(X)+L^{-\omega} g_{R}(X)+\ldots, \\
& X=\left(\beta-\beta_{c}\right) L^{1 / \nu},
\end{aligned}
$$

where $f_{R}(X)$ is a function that is universal up to a multiplicative scale of its argument, $\nu$ is the critical exponent of the correlation length and $\omega$ is the exponent associated with the leading irrelevant operator. By expanding Eq. (48) around $\beta_{c}$, corresponding to $X=0$, we may write

$$
R(\beta, L) \approx R^{*}+\sum_{k=1}^{n} a_{k} X^{k}+L^{-\omega} \sum_{k=0}^{m} b_{k} X^{k}
$$

where, as in Eq. (48), we have neglected next-to-leading scaling corrections. Using this expression it is possible to estimate $\beta_{c}$ and $\nu$ from numerical determinations of $R$.

Since $R_{\xi}$ defined in Eq. (47) is an increasing function of $\beta$, we may write

$$
U(\beta, L)=F_{U}\left(R_{\xi}\right)+O\left(L^{-\omega}\right),
$$

where $F_{U}$ now depends on the universality class only, without any non-universal multiplicative factor. This is true once the boundary conditions and the shape of the lattice have been fixed, provided one uses corresponding quantities in the different models, see, e.g., Ref. 20] and the discussion in Sec.VA, The scaling (151) is particularly convenient to test universality-class predictions, since it permits easy comparisons between different models without any tuning of nonuniversal parameters.

Finally, we also mention that the susceptibility is expected to scale as [8]

$$
\begin{aligned}
\chi(\beta, L) & =L^{2-\eta}\left[f_{\chi}(X)+L^{-\omega} g_{\chi}(X)\right] \\
& =L^{2-\eta}\left[F_{\chi}\left(R_{\xi}\right)+O\left(L^{-\omega}\right)\right],
\end{aligned}
$$

where $f_{\chi}$ and $F_{\chi}$ are universal functions, apart from trivial multiplicative normalizations and a normalization of the argument in the case of $f_{\chi}$.

\section{NUMERICAL RESULTS}

We now present and discuss the results of Monte Carlo (MC) simulations. We use an overrelaxation algorithm, consisting of a combination of heat-bath [44, 45] and microcanonical [46] updates (with ratio 1:5) for the gauge fields (implemented à la Cabibbo-Marinari [47] for $N_{c}>2$ ) and of a combination of Metropolis [48] and microcanonical updates for the scalar field. The Metropolis update was tuned to have an acceptance rate of approximately $30 \%$.

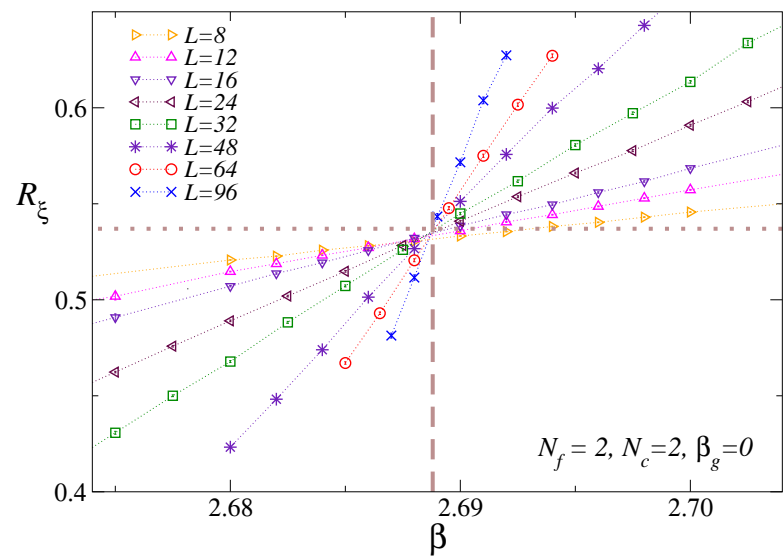

FIG. 1: $R_{\xi}$ versus $\beta$ for $N_{f}=2, N_{c}=2$, and $\beta_{g}=0$. The data for different values of $L$ have a crossing point, whose position provides an estimate of the critical point, $\beta_{c}=2.68885(5)$, indicated by the vertical line. The horizontal line corresponds to the universal value $R_{\xi}^{*}=0.538(1)$ for the $\mathrm{O}(5)$ vector universality class.

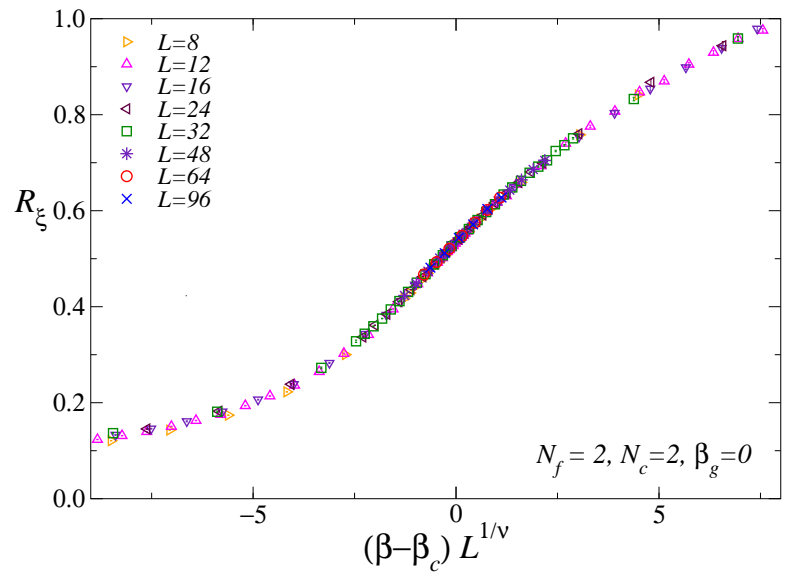

FIG. 2: $R_{\xi}$ versus $\left(\beta-\beta_{c}\right) L^{1 / \nu}$ for $N_{f}=2, N_{c}=2$, and $\beta_{g}=$ 0 . We use $\beta_{c}=2.68885$ and $\nu=0.779$, the estimate of the correlation-length exponent for the $\mathrm{O}(5)$ vector universality class, see Ref. [50].

\section{A. FSS analysis for $N_{f}=2$ and $N_{c}=2$}

In this section we present the numerical results obtained for $N_{f}=2$ and $N_{c}=2$. We start by analyzing the computationally simplest case $\beta_{g}=0$. In this case we performed simulations on lattices of size up to $L=96$.

In Fig. 1 we show the estimates of $R_{\xi}$ for different values of $L$ and $\beta$. They display the typical behavior expected at a continuous transition: Different curves have an approximate crossing point and the slopes increase by increasing the lattice size. Eq. (50) can then be used to extract the critical coupling $\beta_{c}$ and the critical exponent $\nu$. For this purpose we first perform standard nonlinear (unbiased) fits to the ansatz

$$
R_{\xi}=R_{\xi}^{*}+a_{1} X, \quad X=\left(\beta-\beta_{c}\right) L^{1 / \nu},
$$




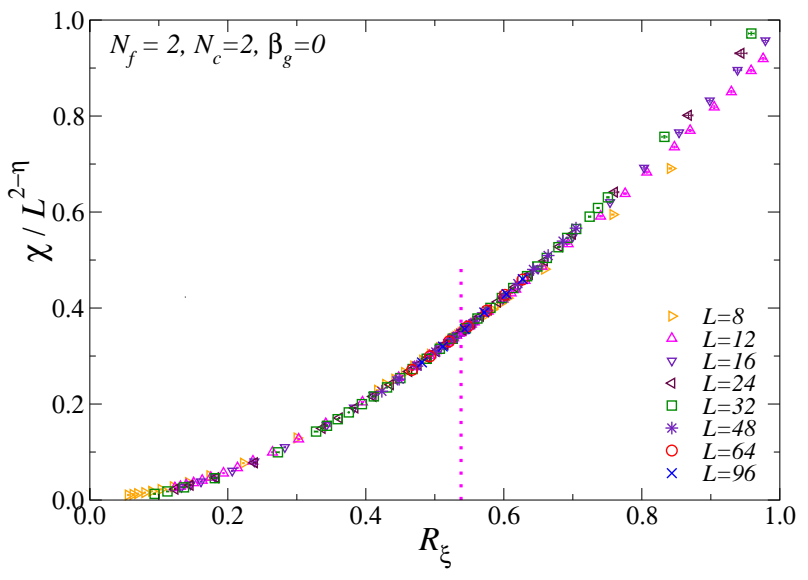

FIG. 3: Rescaled susceptibility $\chi / L^{2-\eta}$ versus $R_{\xi}$, for $N_{f}=2$, $N_{c}=2$, and $\beta_{g}=0$. We use the estimate $\eta=0.034$, the estimate for the $\mathrm{O}(5)$ vector universality class, see Ref. [50]. The dotted vertical line corresponds to the critical value $R_{\xi}^{*}$ for the $\mathrm{O}(5)$ vector universality class.

\begin{tabular}{crcccc}
\hline \hline \multicolumn{1}{c}{$\delta$} & $L_{\min }$ & \multicolumn{1}{c}{$\beta_{c}$} & \multicolumn{1}{c}{$R_{\xi}^{*}$} & $\chi^{2} /$ d.o.f. & \# data \\
\hline 0.05 & 8 & $2.68886(3)$ & $0.5381(3)$ & 1.1 & 45 \\
0.10 & 8 & $2.68887(2)$ & $0.5378(3)$ & 1.4 & 68 \\
0.05 & 12 & $2.68880(4)$ & $0.5372(6)$ & 1.1 & 33 \\
0.10 & 12 & $2.68880(3)$ & $0.5364(5)$ & 1.4 & 52 \\
0.05 & 24 & $2.68886(8)$ & $0.539(3)$ & 1.2 & 13 \\
0.10 & 24 & $2.68884(6)$ & $0.538(2)$ & 1.3 & 26 \\
\hline \hline
\end{tabular}

TABLE I: Results of the biased fits of $R_{\xi}$ to the Ansatz (50) with $n=1, m=0$, fixing $\nu$ and $\omega$ to the $\mathrm{O}(5)$ values reported in Eq. (55). Results for $N_{c}=N_{f}=2$ and $\beta_{g}=0$.

using data within the self-consistent window $R_{\xi}(\beta, L) \in$ $\left[R_{\xi}^{*}(1-\delta), R_{\xi}^{*}(1+\delta)\right]$. For $\delta=0.1$ and $L \geq L_{\min }=16$, we obtain $\beta_{c}=2.68869(2), \nu=0.775(6)$, and $R_{\xi}^{*}=$ $0.5340(2)$, with $\chi^{2} /$ d.o.f. $\approx 1.5$ (30 data, d.o.f. is the number of degrees of freedom of the fit). The exponent $\nu$ is consistent with that of the $\mathrm{O}(5)$ vector universality class, whose universal critical exponents are [49 52 ]

$$
\nu=0.779(3), \quad \eta=0.034(1), \quad \omega=0.79(2) .
$$

To corroborate this identification, we perform biased fits to Eq. (50), with $n=1$ and $m=0$ (we include a single scaling correction term), fixing $\nu$ and $\omega$ to the $\mathrm{O}(5)$ values reported in Eq. (55). Again, we use a self-consistent fit window $R_{\xi}(\beta, L) \in\left[R_{\xi}^{*}(1-\delta), R_{\xi}^{*}(1+\delta)\right]$. The results are reported in Table [1. Our final biased estimates, that take into account the dependence of the fit parameters on $\delta$ and $L_{\min }$, are

$$
\beta_{c}=2.68885(5), \quad R_{\xi}^{*}=0.538(2) .
$$

The errors also take into account the variation of the estimates as $\nu$ and $\omega$ vary within one error bar. The corresponding scaling plot is shown in Fig. 2] where $R_{\xi}$
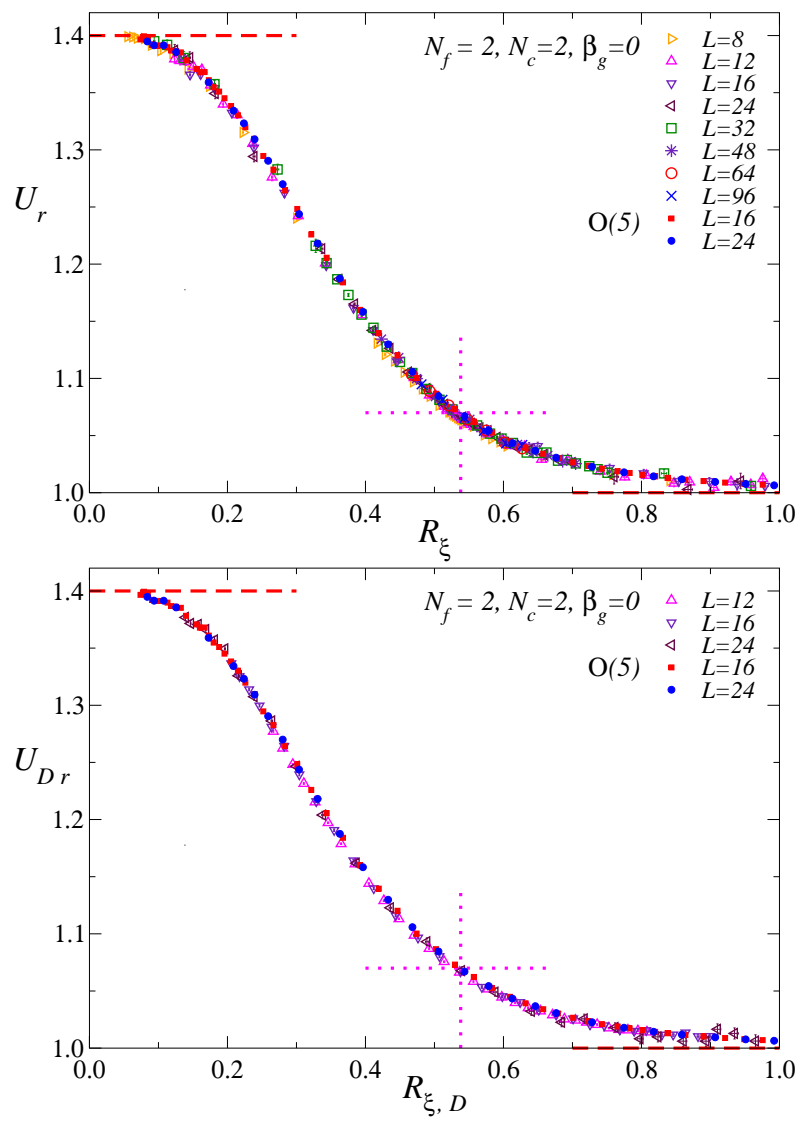

FIG. 4: Rescaled Binder parameter $U_{r}$ versus $R_{\xi}$ (top) and rescaled Binder parameter $U_{D r}$ versus $R_{\xi, D}=\xi_{D} / L$. Results for $N_{f}=2, N_{c}=2$, and $\beta_{g}=0$. Data are in good agreement with the the numerical results for the Binder parameter obtained by numerical simulations of the $\mathrm{O}(5)$ vector lattice model. The dotted horizontal and vertical lines correspond to the universal values $U^{*}=1.069(1)$ and $R_{\xi}^{*}=0.538(1)$ of the $\mathrm{O}(5)$ universality class. The dashed horizontal lines correspond to $U_{r}=7 / 5$ and $U_{r}=1$, the asymptotic values for $R_{\xi} \rightarrow 0$ and for $R_{\xi} \rightarrow \infty$, respectively.

is plotted versus $X=\left(\beta-\beta_{c}\right) L^{1 / \nu}$ using $\beta_{c}=2.68885$ and the $\mathrm{O}(5)$ value $\nu=0.779$. The agreement is excellent. Note also that the estimate of $R_{\xi}^{*}$ is consistent with $R_{\xi}^{*}=0.538(1)$, obtained in the $\mathrm{O}(5)$ vector model using the vector correlation function [50]. Also the behavior of the susceptibility $\chi$ is consistent with a transition in the $\mathrm{O}(5)$ universality class. If we fix $\eta$ to the $\mathrm{O}(5)$ value [see Eq. (55)], the ratio $\chi / L^{2-\eta}$ scales nicely when plotted versus $R_{\xi}$, as expected from the scaling relation Eq. (53), see Fig. 3 ,

Additional evidence that the transition belongs to the $\mathrm{O}(5)$ vector universality class is provided by the analysis of the Binder parameter $U$ defined in Eq. (35). To perform the correct universality check, we should compare corresponding quantities in our model and in the $\mathrm{O}(5)$ vector model. As we discuss at length in App. A, the Binder parameter that corresponds to the $\mathrm{O}(5)$ parameter is $U_{\Gamma}$ defined by using $\mathcal{T}^{\alpha \beta}$, see Eq. (A8). Using 


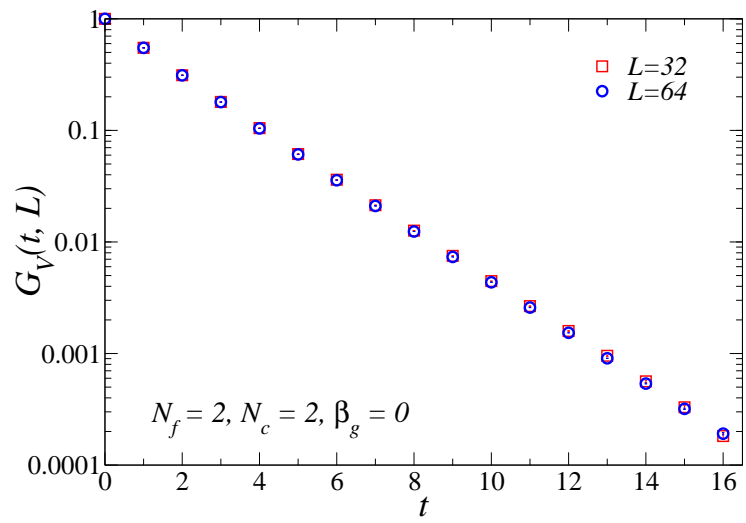

FIG. 5: The correlation function $G_{V}$ defined in Eq. (40), for $N_{f}=2, N_{c}=2$, and $\beta_{g}=0$ at $\beta_{c}$. It shows a large-distance exponential behavior $\sim e^{-x / \xi_{g}}$ with $\xi_{g}=1.92(2)$.

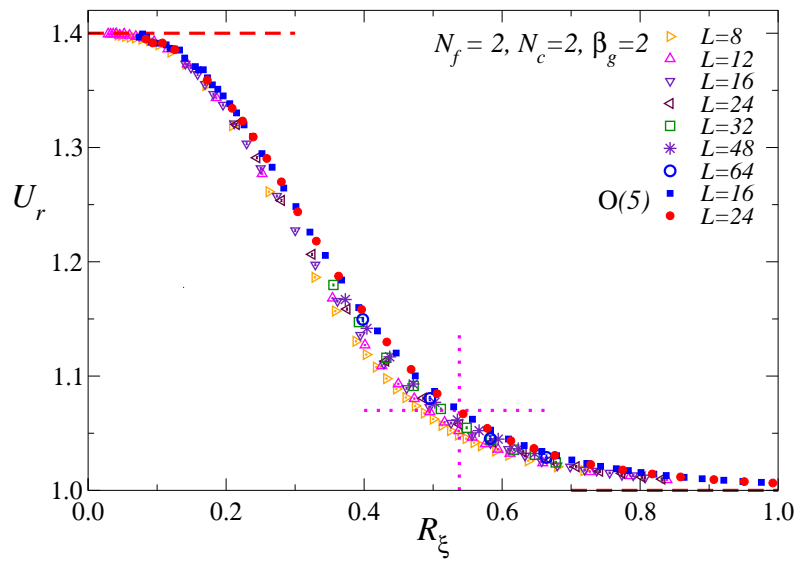

FIG. 6: Estimates of the rescaled Binder parameter $U_{r}$ versus $R_{\xi}$, for $N_{f}=2, N_{c}=2$, and $\beta_{g}=2$ and of the usual Binder parameter for the $\mathrm{O}(5)$ vector model. The dotted horizontal and vertical lines indicate the universal values $U^{*}=1.069(1)$ and $R_{\xi}^{*}=0.538(1)$ of the $\mathrm{O}(5)$ universality class. The dashed horizontal lines correspond to the asymptotic values $U_{r}=7 / 5$ and $U_{r}=1$ for $R_{\xi} \rightarrow 0$ and $R_{\xi} \rightarrow \infty$, respectively.

the $\mathrm{Sp}(2) / \mathrm{O}(5)$ invariance of the theory, one can easily show that $U_{\Gamma}$ and $U$ simply differ by a multiplicative constant, see Eq. A15. Therefore, the renormalized Binder parameter

$$
U_{r}=\frac{21}{25} U
$$

should behave as the vector Binder parameter in the $\mathrm{O}(5)$ vector model. If we perform biased fits to Eq. (50) analogous to those we performed for $R_{\xi}$, we obtain $U_{r}^{*}=$ $1.070(1)$, which is in agreement with the $\mathrm{O}(5)$ estimate $U_{\mathrm{O}(5)}^{*}=1.069(1)$ reported in Ref. [50]. A conclusive evidence for an $\mathrm{O}(5)$ critical behavior is provided by Fig. 4, where we report $U_{r}$ versus $R_{\xi}$ (upper panel). The numerical data fall on top of those obtained in the $\mathrm{O}(5)$ vector model.

As we discussed in Sec. III, in the models with $N_{c}=2$ the $\mathrm{U}(1)$ flavor symmetry breaks at the same $\beta$ where the $\mathrm{SU}\left(N_{f}\right)$ is broken, since the two groups are subgroups of the larger symmetry group $\operatorname{Sp}\left(N_{f}\right)$. To verify this point, we have estimated several observables in terms of the order parameter $D_{x}$ defined in Eq. (36). We have verified that the correlation length $\xi_{D}$ defined using the correlation function (37) is identical, within errors, to $\xi$. Moreover, we have studied the behavior of the Binder parameter $U_{D}$. Again, to obtain a quantity that can be directly related to the $\mathrm{O}(5)$ Binder parameter, we have considered, see Eq. A15),

$$
U_{D r}=\frac{10}{7} U_{D} .
$$

In Fig. 4 (lower panel) we report $U_{D r}$ versus $R_{\xi, D}=$ $\xi_{D} / L$. The data are compared with the $\mathrm{O}(5)$ corresponding data, observing again an excellent agreement.

Finally, we have computed the correlation function $G_{V}(t)$, defined in Eq. (40). As it is evident from Fig. 5, it is short-ranged and essentially independent of $L$ even at the critical point. It has a very clear exponential behavior, $G_{V}(t) \sim \exp \left(-x / \xi_{g}\right)$, with $\xi_{g}=1.92(2)$, independently of the size $L$. We also analyzed the Polyakov loop which is expected to behave as $e^{-L / \xi_{P}}$. The estimates of $\xi_{P}$ are close to those of $\xi_{g}$, but with significantly larger errors.

We have also verified that the analogous results are obtained for $\beta_{g} \neq 0$. For this purpose we performed MC simulations at $\beta_{g}=-2$ (using lattices up to $L=32$ ) and at $\beta_{g}=2$ (using lattices up to $L=48$ ). In both cases data fully support the presence of a continuous transition in the $\mathrm{O}(5)$ universality class. As an example, in Fig. 6] we plot $U_{r}$ versus $R_{\xi}$ for $\beta_{g}=2$. Again, the data fall on top of the corrisponding ones obtained in the $\mathrm{O}(5)$ vector model. Biased fits to Eq. (50) allow us to obtain the estimates $\beta_{c}\left(\beta_{g}=-2\right)=3.794(2)$ and $\beta_{c}\left(\beta_{g}=2\right)=1.767(1)$. While the critical coupling at $\beta_{g}=2$ is significantly lower than the value $\beta_{c}\left(\beta_{g}=0\right) \approx 2.689$, it is still quite larger than the value $\beta_{c}=0.96339(1)$ which is attained in the limit of large $\beta_{g}$, when the model become equivalent to the $\mathrm{O}(8)$ vector model [53]. This could explain the absence of significant crossover effects in our data induced by the unstable $\mathrm{O}\left(2 N_{f} N_{c}\right)$ fixed point at $\beta_{g} \rightarrow \infty$, which have instead been observed in the abelian case [21]. We finally note that the approach to the asymptotic scaling behavior is significantly slower for $\beta_{g}=2$ than for $\beta_{g}=0$, see Fig. 6. This is likely related to the fact that the gauge length scale $\xi_{g}$ at the transition is larger at $\beta_{g}=2$ than at $\beta_{g}=0$. Indeed, we find $\xi_{g}\left(\beta_{g}=2\right)=2.46(4)$, to be compared with $\xi_{g}\left(\beta_{g}=0\right)=1.92(2)$.

The above results provide a robust evidence that the lattice scalar chromodynamics for $N_{f}=2$ and $N_{c}=2$ undergoes a continuous transition in the $\mathrm{O}(5)$ universality class. This result agrees with the predictions of the LGW approach, assuming that the critical behavior is determined by the global symmetry group and that the gauge degrees of freedom are irrelevant. 


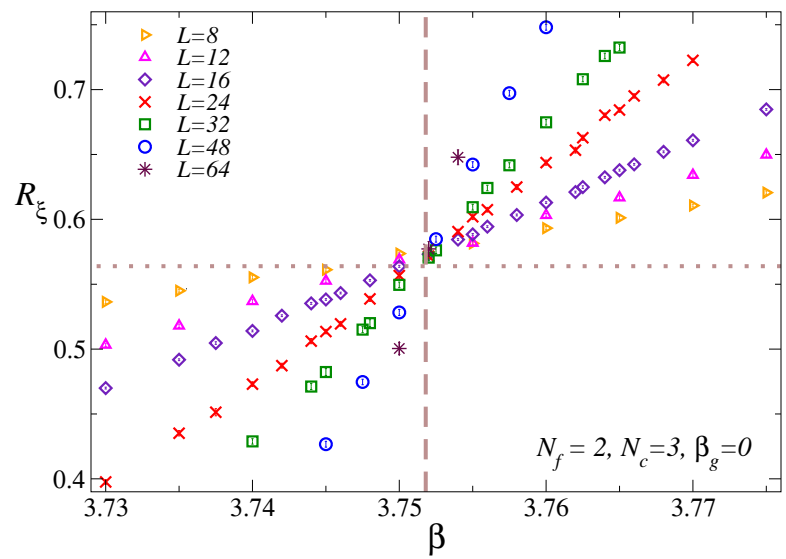

FIG. 7: $\quad R_{\xi}$ versus $\beta$ for $N_{f}=2, N_{c}=3$, and $\beta_{g}=0$. The data for different values of $L$ show a crossing point, whose position provides an estimate of the critical point, $\beta_{c}=3.7518(2)$, indicated by the vertical line. The horizontal line corresponds to the universal value $R_{\xi}^{*}=0.5639(2)$ of the $\mathrm{O}(3)$ vector universality class.

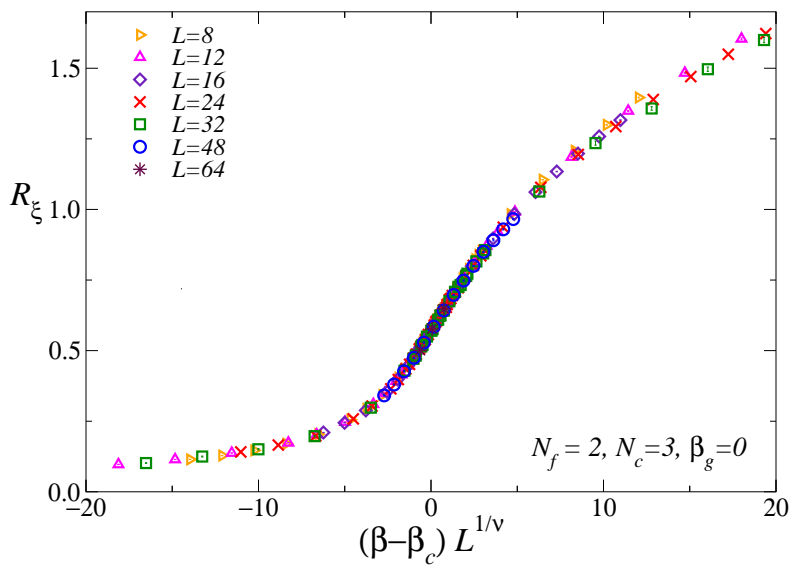

FIG. 8: $\quad R_{\xi}$ versus $\left(\beta-\beta_{c}\right) L^{1 / \nu}$ for $N_{f}=2, N_{c}=3$, and $\beta_{g}=0$. We use $\beta_{c}=3.7518$ and $\nu=0.7117$, the correlationlength exponent for the $\mathrm{O}(3)$ vector universality class.

\section{B. FSS analysis for $N_{f}=2$ and $N_{c}=3,4$}

In this section we consider the model for $N_{f}=2$ and $N_{c}=3,4$. For $N_{c}=3$ and $\beta_{g}=0$ we heve performed simulation up to $L=64$. In Fig. 7 we report $R_{\xi}$ as a function of $\beta$. We observe a crossing point for $\beta \approx 3.75$. To determine the nature of the transition, we again proceed by first performing standard nonlinear (unbiased) FSS fits of the $R_{\xi}$ data to the simplest ansatz Eq. (54), using data within the self-consistent window $R_{\xi}(\beta, L) \in\left[R_{\xi}^{*}(1-\delta), R_{\xi}^{*}(1+\delta)\right]$. For $\delta=0.1$ and $L \geq L_{\min }=8$, we obtain $\beta_{c}=3.7523(1), \nu=0.705(10)$, and $R_{\xi}^{*}=0.5771(5)$, with $\chi^{2} /$ d.o.f. $\approx 1.4$ (28 data). The critical exponent $\nu$ is consistent with that of the $\mathrm{O}(3)$ vector universality class, as predicted by the LGW theory. Indeed, the universal critical exponents and RG invari-

\begin{tabular}{crrlrc}
\hline \hline$\delta$ & $L_{\min }$ & \multicolumn{1}{c}{$\beta_{c}$} & \multicolumn{1}{c}{$R_{\xi}^{*}$} & \multicolumn{2}{c}{$\chi^{2}$ d.o.f. \# data } \\
\hline 0.1 & 8 & $3.75182(9)$ & $0.5673(12)$ & 1.1 & 27 \\
0.1 & 12 & $3.75186(16)$ & $0.569(4)$ & 1.2 & 20 \\
0.1 & 24 & $3.7521(4)$ & $0.577(16)$ & 0.8 & 30 \\
0.1 & 32 & $3.7519(11)$ & $0.57(6)$ & 0.6 & 17 \\
\hline \hline
\end{tabular}

TABLE II: Results of the biased fits for $R_{\xi}$ to Eq. (50) with $n=1$ and $m=2$, fixing $\nu$ and $\omega$ to the $\mathrm{O}(3)$ values reported in Eq. (59). Results for $N_{f}=2, N_{c}=3$, and $\beta_{g}=0$.

ant quantities of the $\mathrm{O}(3)$ universality class which are relevant for our study are [54 56

$$
\begin{aligned}
& \nu=0.7117(5), \eta=0.0378(3), \omega=0.782(13), \\
& R_{\xi}^{*}=0.5639(2), U^{*}=1.1394(3) .
\end{aligned}
$$

Additional evidence for an $\mathrm{O}(3)$ critical behavior is obtained by performing biased fits to Eq. (50) with $n=1$ and $m=0$, fixing $\nu$ and $\omega$ to the $\mathrm{O}(3)$ values reported in Eq. (59). As before, we use data within the self-consistent window $R_{\xi}(\beta, L) \in\left[R_{\xi}^{*}(1-\delta), R_{\xi}^{*}(1+\delta)\right]$. The results are reported in Table [II. The estimates of $R_{\xi}^{*}$ are nicely consistent with the $\mathrm{O}(3)$ estimate $R_{\xi}^{*}=0.5639(2)$. A similar analysis can be done using the Binder parameter $U$. Using $L_{\min }=8$, we obtain the estimates $\beta_{c}=3.7519(2)$ and $U^{*}=1.139(3)$, with $\chi^{2} /$ d.o.f $\approx 1.2$ (27 data). Again, the estimate of $U^{*}$ is in good agreement with the $\mathrm{O}(3)$ value $U^{*}=1.1394(3)$. Our final estimate of the critical temperature, obtained by considering the various systematic errors, is

$$
\beta_{c}=3.7518(2) .
$$

In Figs. 8, 9, and 10 we show different scaling plots that clearly confirm that the transition belongs to the $\mathrm{O}(3)$ universality class. In particular, the data of $U$ plotted versus $R_{\xi}$, see Fig. 9, are nicely consistent with the results obtained in numerical simulations of the $\mathrm{O}(3)$ vector model.

As in the two color case, we have also checked that the above results extend to nonvanishing values of $\beta_{g}$. In particular, simulations have been performed for a few values of $\beta_{g}$ between -9 and 6 . In all cases, the FSS behavior of $U$ as a function of $R_{\xi}$ supports the $\mathrm{O}(3)$ nature of the transition, as can be seen in Fig. [11, where we report the results for $\beta_{g}=-6$ and $\beta_{g}=6$. Again these results are far from trivial, since the critical coupling $\beta_{c}\left(\beta_{g}\right)$ changes from approximately 4.39 to 2.55 as we vary $\beta_{g}$ in the interval $[-9,6]$ (see Fig. 12). Therefore, the effect of $\beta_{g}$ on the dynamics of the system is large. Also the average gauge energy $E_{g}$ at criticality changes significantly. It varies approximately from -0.23 to 0.51 . These values are however still far from the asymptotic values \pm 1 at $\beta_{g} \rightarrow \pm \infty$ and this could explain the absence of sizable crossover effects in our data. This is also consistent with the fact that the correlation length associated with the gauge modes increases with increasing 


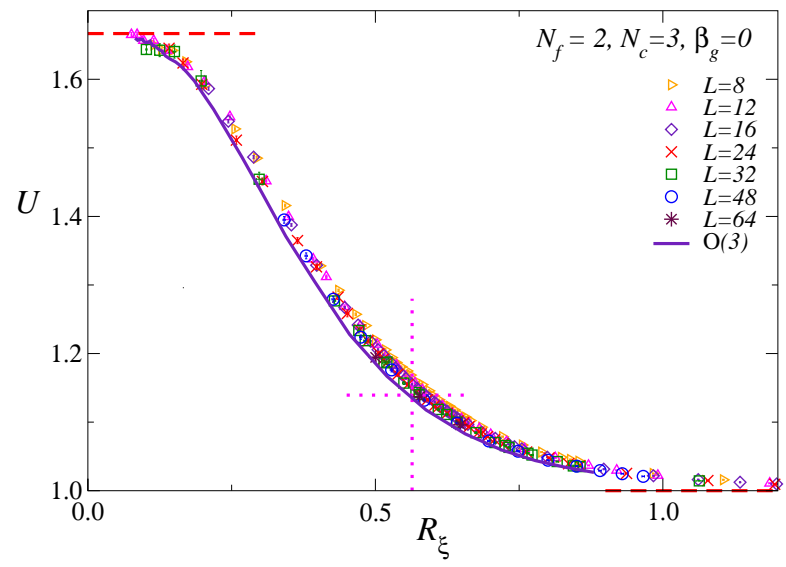

FIG. 9: The Binder parameter $U$ versus $R_{\xi}$, for $N_{f}=2$, $N_{c}=3$, and $\beta_{g}=0$. The data clearly converge to the $\mathrm{O}(3)$ vector universal curve (continuous curve). The dotted horizontal and vertical lines correspond to the universal values $U^{*}=1.1394(3)$ and $R_{\xi}^{*}=0.5639(2)$ of the $\mathrm{O}(3)$ universality class. The dashed horizontal lines correspond to $U=5 / 3$ and $U=1$, the asymptotic values of $R_{\xi} \rightarrow 0$ and for $R_{\xi} \rightarrow \infty$, respectively.

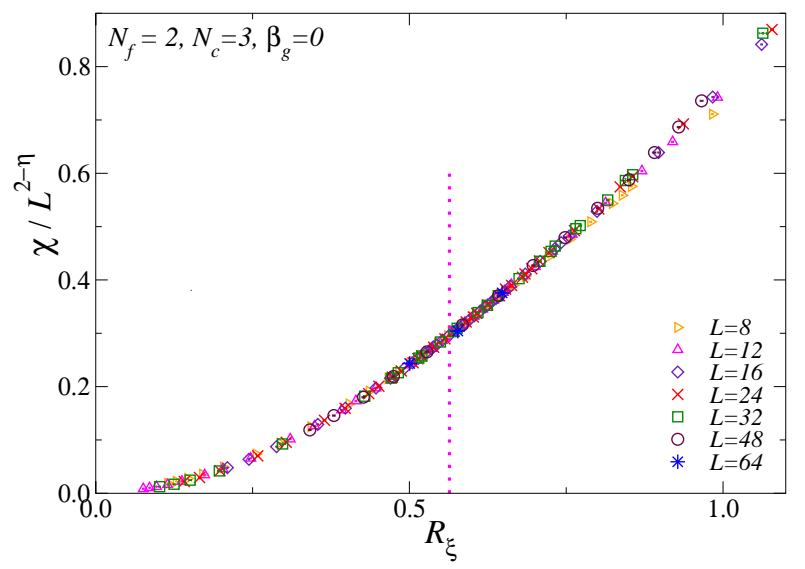

FIG. 10: The rescaled susceptibility $\chi / L^{2-\eta}$ with $\eta=0.0378$, the exponent value in the $\mathrm{O}(3)$ vector universality class, versus $R_{\xi}$, for $N_{f}=2, N_{c}=3$, and $\beta_{g}=0$. The dotted vertical line corresponds to $R_{\xi}^{*}$.

$\beta_{g}$, but nevertheless stays quite small: at the transition we obtain $\xi_{g}\left(\beta_{g}=0\right)=1.60(2), \xi_{g}\left(\beta_{g}=3\right)=1.70(2)$, and $\xi_{g}\left(\beta_{g}=6\right)=2.02(2)$.

As a final check that, for $N_{f}=2$ and any $N_{c} \geq 3$, the transition always belongs to the $\mathrm{O}(3)$ universality class, we performed $\mathrm{MC}$ simulations for $N_{c}=4$ and $\beta_{g}=0$. Also in this case the data of $U$ plotted versus $R_{\xi}$ (we have results for $L \leq 48$ ) clearly approach the $\mathrm{O}(3)$ curve as $L$ is increased, as it can be seen in Fig. (13). Again, the results confirm the LGW predictions.
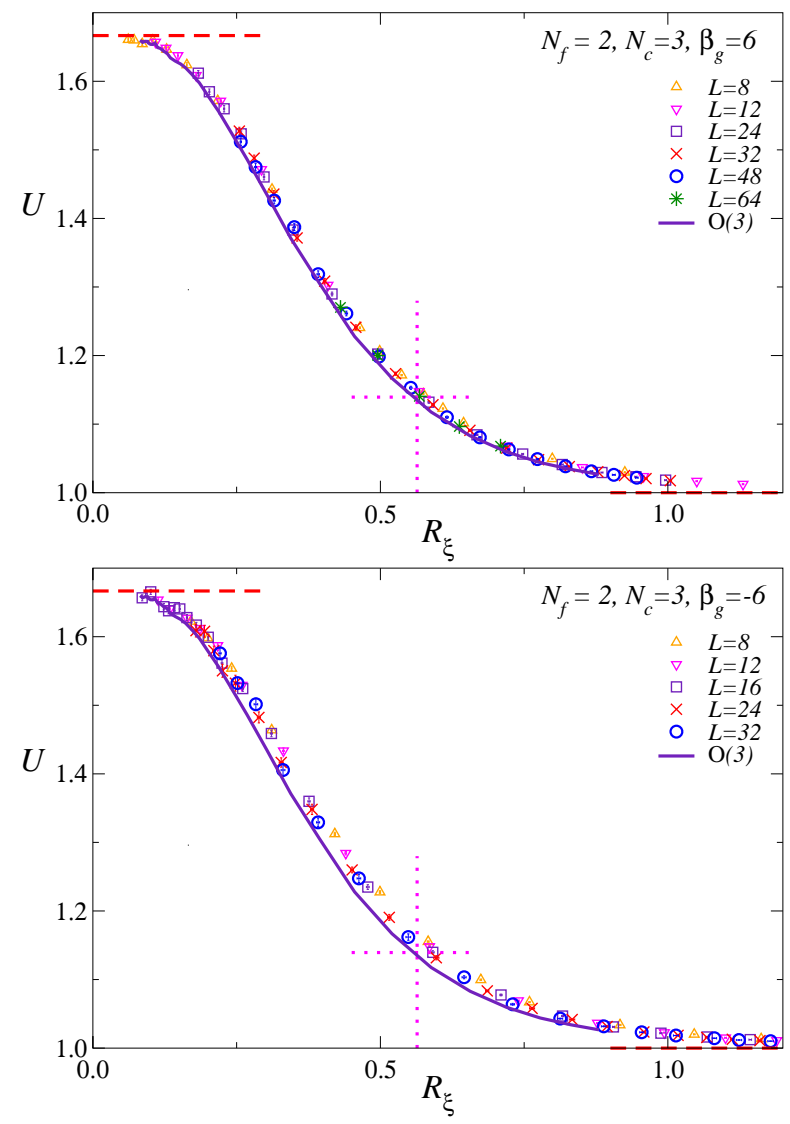

FIG. 11: The Binder parameter $U$ versus $R_{\xi}$, for $N_{f}=2$, $N_{c}=3$. In the lower panel we report results for $\beta_{g}=-6$ up to $L=32$, in the upper panel results for $\beta_{g}=6$ up to $L=64$. The data appear to converge to the $\mathrm{O}(3)$ vector universal curve (continuous line). The dotted horizontal and vertical lines correspond to the universal values $U^{*}=1.1394(3)$ and $R_{\xi}^{*}=0.5639(2)$ of the $\mathrm{O}(3)$ universality class. The dashed horizontal lines correspond to $U=5 / 3$ and $U=1$, the asymptotic values for $R_{\xi} \rightarrow 0$ and $R_{\xi} \rightarrow \infty$.

\section{FSS analysis for $N_{f}=3$}

For $N_{f}=3$ the LGW effective field theory predicts a first-order phase transition for any number of colors. To verify the prediction, we perform sumulations for $N_{c}=2$ and $N_{c}=3$, fixing always $\beta_{g}=0$.

A standard technique to identify first-order phase transitions consists in checking if the maximum value of the susceptibility or of the specific heat scales linearly with the volume. However, for weak first order transitions such a technique is, in practice, quite often ineffective: The values of $L$ at which such a behavior sets in are far larger than those at which simulations can be performed. This is indeed what happens, as we discuss below, for $N_{c}=2$ and 3 .

In Fig. 14 we report the specific heat $C_{V}$ defined in Eq. (30) for $N_{c}=2$. It is clear that the specific heat is apparently diverging as $L$ increases. This allows us to conclude that the transition, if continuous, does not 


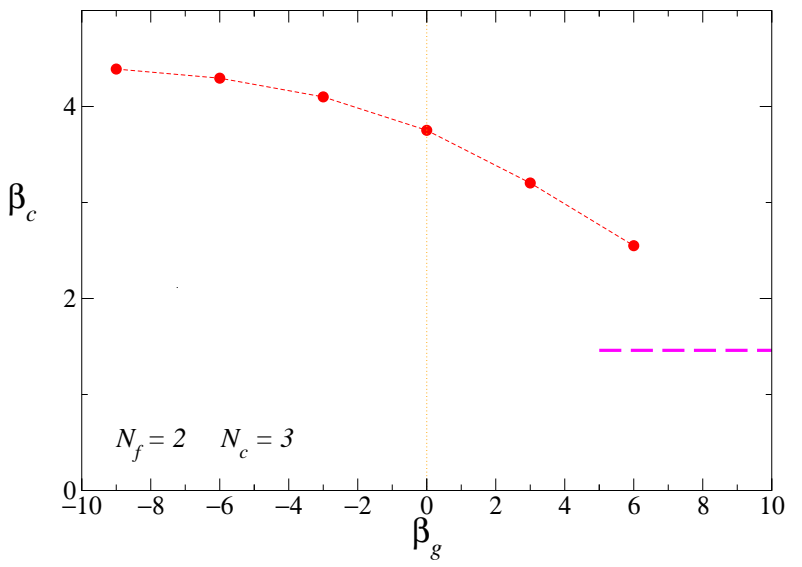

FIG. 12: Estimates of $\beta_{c}$ versus $\beta_{g}$ for the model with $N_{f}=$ $2, N_{c}=3$. The dashed line indicates the critical value in the limit $\beta_{g} \rightarrow \infty$, corresponding to the critical point of the $\mathrm{O}(12)$ theory, $\beta_{c} \approx 1.46$, obtained using the results reported in Ref. [57]. The dotted line connecting the data is drawn to guide the eyes.

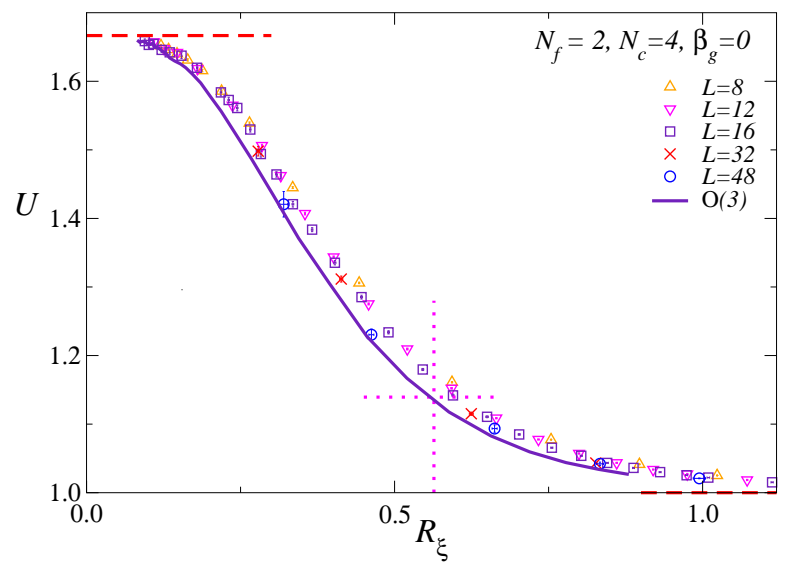

FIG. 13: The Binder parameter $U$ versus $R_{\xi}$, for $N_{f}=2$, $N_{c}=4$, and $\beta_{g}=0$. The data appear to converge to the $\mathrm{O}(3)$ vector universal curve (continuous line). The dotted horizontal and vertical lines correspond to the universal values $U^{*}=1.1394(3)$ and $R_{\xi}^{*}=0.5639(2)$ of the $\mathrm{O}(3)$ universality class. The dashed horizontal lines correspond to $U=5 / 3$ and $U=1$, the asymptotic values for $R_{\xi} \rightarrow 0$ and $R_{\xi} \rightarrow \infty$.

belong to a universality class characterized by a negative value of the critical exponent $\alpha$, like, e.g., the standard $\mathrm{O}(M)$ universality classes for any $M \geq 2[8]$.

In the case of weak first-order transitions, a more useful quantity is the Binder parameter $U$. At a first-order transition, the maximum $U_{\max }$ of $U$ behaves as [58, 59]

$$
U_{\max }=a V\left[1+O\left(V^{-1}\right)\right] .
$$

On the other hand, at a continuous phase transition, $U$ is bounded as $L \rightarrow \infty$. At the critical point $U$ converges to a universal value $U^{*}$, while the data of $U$ corresponding to different values of $R_{\xi}$ collapse onto a common scaling curve as the volume is increased. Therefore, $U$ has

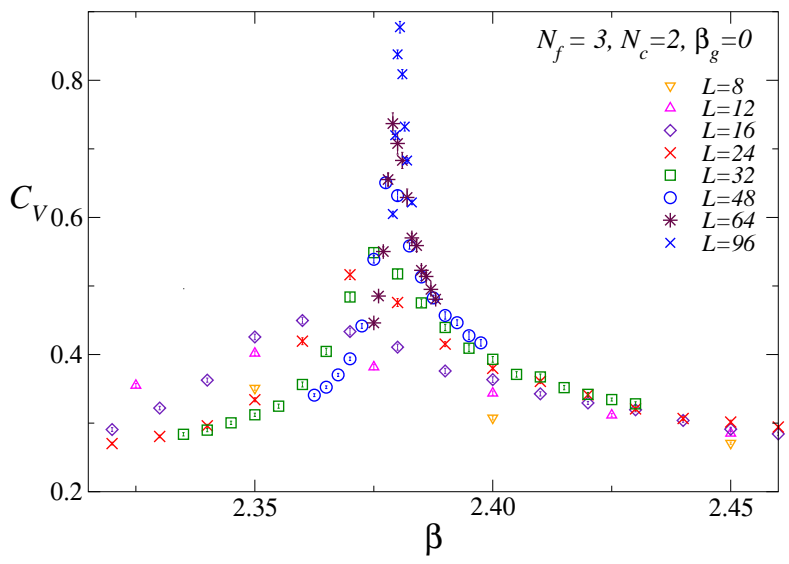

FIG. 14: The specific heat defined in Eq. (30) versus $\beta$ for $N_{c}=2, N_{f}=3$, and $\beta_{g}=0$.

a qualitatively different scaling behavior for first- and second-order transitions. In practice, a first-order transition can be simply identified by verifying that $U_{\max }$ increases with $L$, without the need of explicitly observing the linear behavior in the volume. A second indication of a first-order transition is provided by the plot of $U$ versus $R_{\xi}$. The absence of a data collapse is an early indication of the first-order nature of the transition, as already advocated in Ref. [20]. In Fig. 15] we plot the Binder parameter $U$ versus $R_{\xi}$, for $N_{c}=2$ and $N_{c}=3$, respectivele, and $\beta_{g}=0$. In neither of the two cases an acceptable collapse is obtained and the data display a pronounced peak whose height increases with increasing volume. We take the absence of scaling as an evidence that the transition is not continuous, thus that it is of first order in both cases.

We have also investigated the behavior of the observables related to the breaking of the U(1) flavor symmetry. In Fig. 16 we report the correlation length $\xi_{D}$ and the Binder parameter $U_{D}$, defined in Sec. IV A. Our numerical results show that the correlation length $\xi_{D}$ is always small, even at the transition point $\beta \approx 3.415$, a clear indication that the $\mathrm{U}(1)$ flavor symmetry does not break. The results for the Binder parameter are completely consistent: $U_{D}$ is always compatible with the high-temperature value $U_{D}=2$.

\section{CONCLUSIONS}

In this work we have studied the finite-temperature critical behavior of the lattice multiflavor chromodynamics model defined by the action, Eq. (6). This model is characterized by the presence of a $\mathrm{SU}\left(N_{c}\right)$ gauge symmetry and of a $\mathrm{U}\left(N_{f}\right)$ or $\operatorname{Sp}\left(N_{f}\right)$ global symmetry, depending whether $N_{c} \geq 3$ or $N_{c}=2$. In all cases, we find that the system undergoes a finite-temperature phase transition associated with the condensation of a gaugeinvariant order parameter: the operator $Q_{\boldsymbol{x}}^{a b}$ for $N_{c} \geq 3$ 

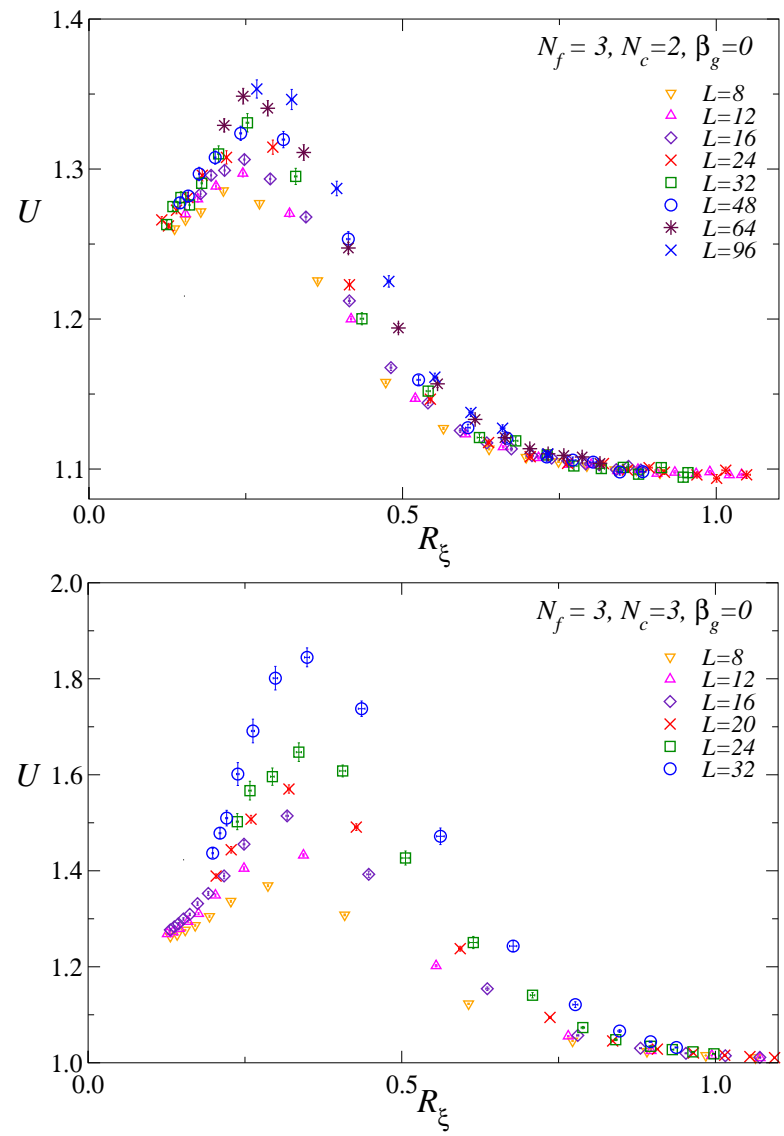

FIG. 15: The Binder parameter $U$ versus $R_{\xi}$, for $N_{f}=3$, $N_{c}=2$ (top) and $N_{c}=3$ (bottom), and $\beta_{g}=0$. The presence of a maximum of $U$ diverging in the large- $L$ limit is a peculiar feature of the behavior at first-order transitions, see, e.g., Refs. [20, 58, 59].

and the operator $\mathcal{T}_{\boldsymbol{x}}^{\alpha \beta}$ for $N_{c}=2$. At the phase transition the global symmetry $\mathrm{SU}\left(N_{f}\right)$ or $\mathrm{Sp}\left(N_{f}\right)$ is spontaneously broken.

To investigate the possible influence of the gauge degrees of freedom on the critical behavior of the model, we determine the universality class of the transition for several values of the number of colors $N_{c}$ and of the numbers of flavors $N_{f}$, also varying the plaquette-coupling coefficient $\beta_{g}$. In the two-flavor case, we always observe a continuous phase transition, in the $3 \mathrm{D} \mathrm{O}(5)$ universality class for $N_{c}=2$ and in the $3 \mathrm{D} \mathrm{O}(3)$ universality class for $N_{c}=3,4$. For $N_{f}=3$ we instead find results compatible with the presence of a first-order phase transition both for $N_{c}=2$ and 3 .

These results agree with the predictions of a LGW analysis based on a gauge-invariant order parameter [36], and therefore indicate the irrelevance of the nonabelian gauge degrees of freedom at the finite-temperature transition. In other words, gauge invariance does not play any role at the transition, apart from that of restricting the fields to the coset $\mathrm{O}(M) / \mathrm{SU}\left(N_{c}\right)$ where $M=2 N_{c} N_{f}$. Such a conclusion is also consistent with the observed be-
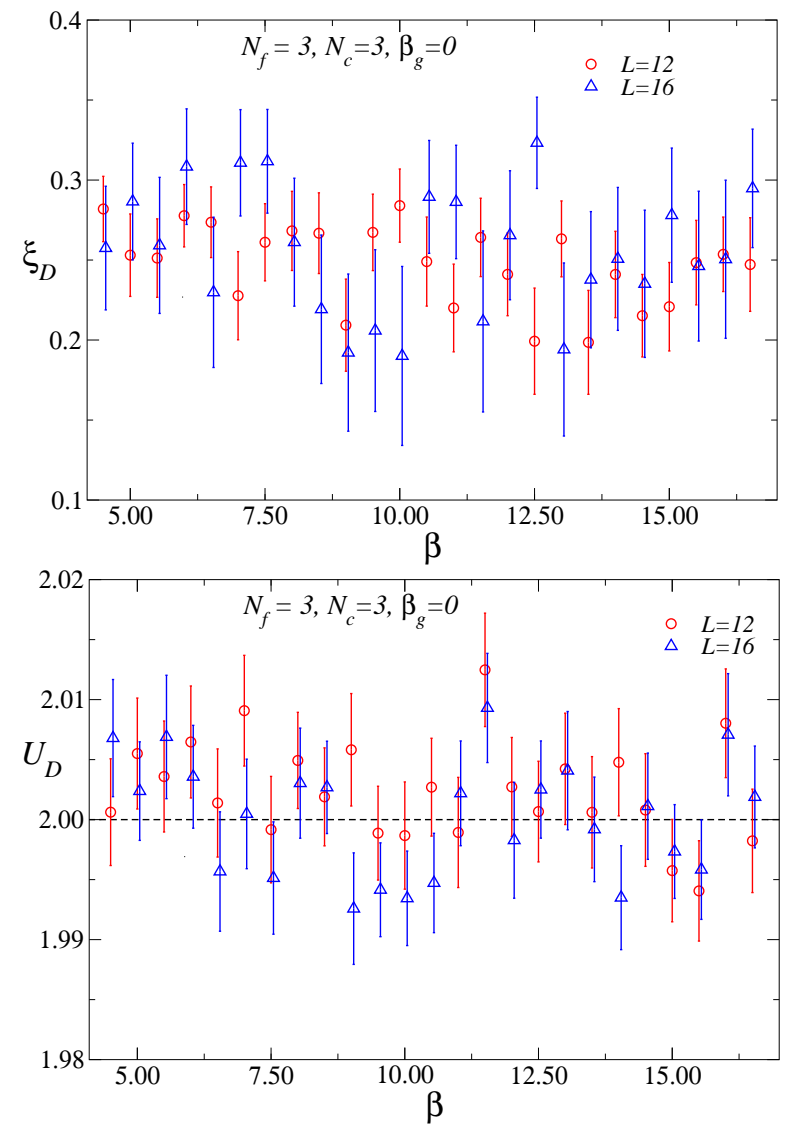

FIG. 16: The correlation length $\xi_{D}$ and the Binder parameter $U_{D}$ versus $\beta$, for $N_{f}=3, N_{c}=3$, and $\beta_{g}=0$. We report results for $\beta$ in the range $[4,17]$. The first-order transition occurs at $\beta_{c} \approx 3.415$.

havior of the correlation function $G_{V}$, defined in Eq. (40), which directly involves the gauge degrees of freedom. In all cases, this correlation function is short-ranged at the transition.

These results strongly support the procedure initially advocated by Pisarski and Wilczek in Ref. [12] to study the chiral phase transition in massless QCD, which makes use of gauge-invariant order parameters to analyze the critical behavior of gauge theories when a global symmetry gets spontaneously broken.

We finally note that there are still several points which deserve to be further investigated. For example, in this work we concentrated on the gauge theory that is obtained by starting from a maximally symmetric $\mathrm{O}(M)$ invariant model and by fixing $\operatorname{Tr} Z_{\boldsymbol{x}}^{\dagger} Z_{\boldsymbol{x}}=1$. It would be interesting to investigate what happens if one or both of these conditions are relaxed. It would also be interesting to study theories with different global and local symmetries that are different from the ones considered in this work.

Acknowledgement Numerical simulations have been performed on the CSN4 cluster of the Scientific Com- 
puting Center at INFN-PISA. We thank Daniele Teresi and Omar Zanusso for useful discussions.

\section{Appendix A: Symplectic observables for $N_{c}=2$}

For $N_{c}=2$ the order parameter is the symplectic analogue of $Q_{\boldsymbol{x}}$ defined in Eq. (28). It is a $2 N_{f} \times 2 N_{f}$ hermitian traceless matrix, which satisfies the relation

$$
J \overline{\mathcal{T}} J+\mathcal{T}=0,
$$

which follows from Eq. (16). It is thus an element of the $\mathfrak{s p}\left(N_{f}\right)$ algebra [41]. It can be parametrized in the block form

$$
\mathcal{T}=\left(\begin{array}{ll}
A_{1} & A_{2} \\
A_{3} & A_{4}
\end{array}\right),
$$

where $A_{1}, A_{2}, A_{3}$ and $A_{4}$ are $N_{f} \times N_{f}$ matrices, $A_{1}$ is hermitian and traceless, $A_{3}$ is antisymmetric and

$$
A_{4}=\bar{A}_{1}, \quad A_{3}=-\bar{A}_{2} .
$$

It is not difficult to show that $\mathcal{T}$ can be expressed in terms of the two order parameters $Q_{\boldsymbol{x}}^{f g}$ and $D_{\boldsymbol{x}}^{f g}$. Indeed, we have

$$
A_{1}=Q, \quad A_{3}=-D .
$$

This result implies that the critical behavior encoded in $\mathcal{T}_{\boldsymbol{x}}^{\alpha \beta}$ can be also investigated by studying $Q_{x}^{f g}$. However, some care should be exercised, when comparing the results with the $\operatorname{Sp}\left(N_{f}\right)$ predictions. We define the correlation function of the $\mathcal{T}$ field:

$$
G_{\Gamma}(\boldsymbol{x}-\boldsymbol{y})=\left\langle\operatorname{Tr} \mathcal{T}_{\boldsymbol{x}} \mathcal{T}_{\boldsymbol{y}}\right\rangle
$$

Such a correlation can be related to the correlation of the $Q$ field defined in Eq. (32). We use the relation

$$
\begin{aligned}
& \left\langle\mathcal{T}_{\boldsymbol{x}}^{\alpha \beta} \mathcal{T}_{\boldsymbol{y}}^{\gamma \delta}\right\rangle=\frac{1}{2\left(N_{f}-1\right)\left(2 N_{f}+1\right)} G_{\Gamma}(\boldsymbol{x}-\boldsymbol{y}) \\
& \quad \times\left(J^{\alpha \gamma} J^{\beta \delta}+\delta^{\alpha \delta} \delta^{\beta \gamma}-\frac{1}{N_{f}} \delta^{\alpha \beta} \delta^{\gamma \delta}\right)
\end{aligned}
$$

which follows from the $\operatorname{Sp}\left(N_{f}\right)$ invariance of the theory. We obtain the relation

$$
G_{\Gamma}(\boldsymbol{x})=\frac{2\left(2 N_{f}+1\right)}{\left(N_{f}+1\right)} G(\boldsymbol{x}) .
$$

It implies that, if one uses Eq. (34), the same correlation length is obtained from $G_{\Gamma}(\boldsymbol{x})$ or $G(\boldsymbol{x})$. The behavior of the Binder parameter is more involved. For $N_{c}=2$ the natural Binder parameter is

$$
U_{\Gamma}=\frac{\left\langle\nu_{2}^{2}\right\rangle}{\left\langle\nu_{2}\right\rangle^{2}}, \quad \nu_{2}=\frac{1}{V^{2}} \sum_{\boldsymbol{x} \boldsymbol{y}} \operatorname{Tr} \mathcal{T}_{\boldsymbol{x}} \mathcal{T}_{\boldsymbol{y}} .
$$

In general such a quantity is not related to $U$ defined in Eq. (35), except for $N_{f}=2$, as we discuss below.

For $N_{f}=2$ the global invariance group is isomorphic to $\mathrm{SO}(5)$. It is useful to make this correspondence explicit. We can rewrite the blocks $A_{1}$ and $A_{3}$ in Eq. (A2) as

$$
\begin{aligned}
& A_{1}=\left(\begin{array}{cc}
\phi_{3} & \phi_{1}-i \phi_{2} \\
\phi_{1}+i \phi_{2} & -\phi_{3}
\end{array}\right), \\
& A_{3}=\left(\begin{array}{cc}
0 & \phi_{4}+i \phi_{5} \\
-\phi_{4}-i \phi_{5} & 0
\end{array}\right) .
\end{aligned}
$$

Since

$$
\left(\mathcal{T}^{2}\right)^{\alpha \beta}=\frac{1}{4} \delta^{\alpha \beta},
$$

we can verify that

$$
\sum_{i=1}^{5} \phi_{i}^{2}=1
$$

Moreover, one can easily verify that, under infinitesimal $\operatorname{Sp}(2)$ transformations, the vector $\left(\phi_{1}, \ldots, \phi_{5}\right)$ transforms as an $\mathrm{SO}(5)$ vector. Thus, the redefinition $\mathcal{T} \rightarrow \phi$ explicitly realizes the isomorphism between $\mathrm{Sp}(2) / \mathbb{Z}_{2}$ and $\mathrm{SO}(5)$. Since

$$
\begin{aligned}
& \operatorname{Tr} Q_{\boldsymbol{x}} Q_{\boldsymbol{y}}=2 \sum_{a=1}^{3} \phi_{\boldsymbol{x}}^{a} \phi_{\boldsymbol{y}}^{a}, \\
& \bar{D}_{\boldsymbol{x}} D_{\boldsymbol{y}}=\sum_{a=4}^{5} \phi_{\boldsymbol{x}}^{a} \phi_{\boldsymbol{y}}^{a}
\end{aligned}
$$

we obtain the relations

$$
\begin{aligned}
G_{\Gamma}(\boldsymbol{x}-\boldsymbol{y}) & =4\left\langle\phi_{\boldsymbol{x}} \cdot \boldsymbol{\phi}_{\boldsymbol{y}}\right\rangle, \\
G(\boldsymbol{x}-\boldsymbol{y}) & =\frac{3}{20} G_{\Gamma}(\boldsymbol{x}-\boldsymbol{y}), \\
G_{D}(\boldsymbol{x}-\boldsymbol{y}) & =\frac{1}{10} G_{\Gamma}(\boldsymbol{x}-\boldsymbol{y}),
\end{aligned}
$$

where we have used the $\mathrm{O}(5)$ symmetry of the theory. For the Binder parameters we have

$$
U_{\Gamma}=\frac{\left\langle\nu_{2 \phi}^{2}\right\rangle}{\left\langle\nu_{2 \phi}\right\rangle^{2}}, \quad \nu_{2 \phi}=\frac{1}{V^{2}} \sum_{\boldsymbol{x} \boldsymbol{y}}\left\langle\boldsymbol{\phi}_{\boldsymbol{x}} \cdot \boldsymbol{\phi}_{\boldsymbol{y}}\right\rangle,
$$

which shows that $U_{\Gamma}$ corresponds to the usual $\mathrm{O}(5)$ Binder parameter, and

$$
U=\frac{25}{21} U_{\Gamma}, \quad U_{D}=\frac{10}{7} U_{\Gamma} .
$$

\section{Appendix B: Symplectic Landau-Ginzburg-Wilson theory for $N_{c}=2$}

To define the LGW theory for $N_{c}=2$, we introduce a coarse-grained continuum analogue $\Psi$ of $\mathcal{T}$, which satisfies the condition

$$
J \bar{\Psi} J+\Psi=0 .
$$


The corresponding action is given in Eq. (22). For $N_{f}=$ 2, we obtain the $\mathrm{O}(5) \mathrm{LGW}$ model. Indeed, in this case we can set

$$
\begin{aligned}
& A_{1}=\left(\begin{array}{cc}
\psi_{3} & \psi_{1}-i \psi_{2} \\
\psi_{1}+i \psi_{2} & -\psi_{3}
\end{array}\right), \\
& A_{3}=\left(\begin{array}{cc}
0 & \psi_{4}+i \psi_{5} \\
-\psi_{4}-i \psi_{5} & 0
\end{array}\right),
\end{aligned}
$$

from which it easily follows that

$$
\Psi^{2}=I\left(\sum_{i=1}^{5} \psi^{2}\right), \quad \operatorname{Tr} \Psi^{3}=0,
$$

and thus the LGW effective theory for $\Psi$ in the $\operatorname{Sp}(2)$ case is equivalent to that for the $\mathrm{O}(5)$ vector model.

\section{Appendix C: The behavior for $\beta \rightarrow \infty$}

In this appendix we study the large- $\beta$ limit of the model described by the action $S_{g}$, Eq. (6). As the system is ferromagnetic, the global minimum of the $\beta$-dependent part of the action is obtained by minimizing the contribution of each link. This is obtained by setting

$$
Z_{\boldsymbol{x}}=U_{\boldsymbol{x}, \mu} Z_{\boldsymbol{x}+\hat{\mu}}
$$

on each link. This relation implies that

$$
Q_{\boldsymbol{x}}=Q_{\boldsymbol{x}+\hat{\mu}}, \quad D_{\boldsymbol{x}}=D_{\boldsymbol{x}+\hat{\mu}}
$$

on each link, where $Q_{\boldsymbol{x}}$ and $D_{\boldsymbol{x}}$ are the order parameters defined in Eqs. (21) and (26). The unit-length condition implies that $Q_{\boldsymbol{x}}$ is nonvanishing: the system is fully ordered in the limit $\beta \rightarrow \infty$ and therefore the $S U\left(N_{f}\right)$ subgroup is broken at zero temperature. As for the U(1) order parameter $D_{\boldsymbol{x}}$, we shall show below that $D_{\boldsymbol{x}}$ is nonvanishing for $N_{c}=2$. This is obvious as the $\mathrm{U}(1)$ subgroup is a subgroup of the $\operatorname{Sp}\left(N_{f}\right)$ symmetry group, which is broken. On the other hand, for $N_{c} \geq 3$, we find $D_{\boldsymbol{x}}=0$ : the $\mathrm{U}(1)$ flavor symmetry is not broken.

Let us now consider any closed path $C_{\boldsymbol{x}}$ that starts and ends in the same point $\boldsymbol{x}$. By repeated applications of condition (C1), we obtain the consistency condition

$$
Z_{\boldsymbol{x}}^{a f}=\sum_{b}\left[\prod_{l \in C_{\boldsymbol{x}}} U_{l}\right]^{a b} Z_{\boldsymbol{x}}^{b f}
$$

This relation implies that the product of the links along the path has at least one unit eigenvalue. For an $\mathrm{SU}(2)$ matrix, this implies that the product is the identity matrix. Therefore, for $N_{c}=2$, we can set $U_{\boldsymbol{x}, \mu}=1$ modulo gauge transformations. For $N_{c} \geq 3$, we obtain the condition

$$
\prod_{l \in C_{\boldsymbol{x}}} U_{l}=V_{\boldsymbol{x}}^{\dagger} W V_{\boldsymbol{x}}
$$

with $V_{\boldsymbol{x}} \in \mathrm{SU}\left(N_{c}\right)$ and

$$
W=\left(\begin{array}{cc}
\widehat{W} & 0 \\
0 & 1
\end{array}\right)
$$

where $\widehat{W}$ is an $\mathrm{SU}\left(N_{c}-1\right)$ matrix. If $\widehat{W}$ does not have unit eigenvalues (this is the case for a generic unitary matrix), then

$$
Z_{\boldsymbol{x}}=V_{\boldsymbol{x}}^{\dagger} A, \quad A=\left(\begin{array}{l}
0 \\
\hat{z}
\end{array}\right),
$$

where $A$ is an $N_{c} \times N_{f}$ matrix such that $A_{i j}=0$ for any $i=1, \ldots N_{c}-1 ; \hat{z}$ is a unit vector of $N_{f}$ elements.

To obtain more information on the gauge configurations relevant for $\beta \rightarrow \infty$ we have performed simulations for $\beta_{g}=0$ on small lattices $\left(2^{3}\right.$ and $\left.4^{3}\right)$ for very large $\beta$ values (from $\beta=50$ up to $\beta=300$ ) and then we have extrapolated the results to $\beta \rightarrow \infty$. Results for different quantities are reported in Tables III and IV. Note that we are indeed probing the system in the large $\beta$ regime as the average energy $E$ defined in Eq. (30) converges to -3 . In Tables III and IV we also report the average gauge energy defined in Eq. (31). For $N_{c}=2$, results are consistent with the plaquette being the identity matrix. For $N_{c} \geq 3$, data for the average gauge energy, cf. Eq. (31), are consistent with

$$
E_{g}=\frac{1}{N_{c}}
$$

Note that this is not an exact equality for finite $L$. However, deviations decrease as $L$ increases from 2 to 4 . Such a result can be explained by assuming that the relevant configurations are such that all plaquettes can be rewritten in the form (C4), where $W$ is given in Eq. (C5). Indeed, if this is the case and $\widehat{W}$ is randomly distributed, we obtain the result (C7). Of course, we are not claiming that all minimizing configurations are such that Eq. (C5) and (C6) hold. We only claim that the number of these configurations is exponentially larger in the lattice volume than the others, so that they dominate the effective asymptotic behavior. As a check, we have determined the average of $P^{2}$, where $P_{\boldsymbol{x}}^{f g}$ is defined by

$$
P_{\boldsymbol{x}}^{f g}=\sum_{a} \bar{Z}_{\boldsymbol{x}}^{a f} Z_{\boldsymbol{x}}^{a g} .
$$

In general, such an operator is not a projector, i.e., $P^{2} \neq$ $P$. However, if the $Z$ fields satisfy Eq. (C6), we have $P^{2}=P$ and in particular $\operatorname{Tr} P^{2}=1$. The results reported in Tables III and IV are in perfect agreement with this result, confirming the above analysis.

If the relevant configurations have the form (C6) it is immediate to prove that $D_{\boldsymbol{x}}=0$ everywhere. The U(1) flavor symmetry is not broken at $\beta=\infty$, at least for $\beta_{g}=0$. It is easy to understand under which conditions the order parameter $D_{\boldsymbol{x}}$ is not zero. If we imagine the 
field $Z^{a f}$ as a collection of $N_{f}$ complex vectors of dimension $N_{c}$, then $D_{\boldsymbol{x}}$ does not vanish if $N_{c}$ of these vectors are nonvanishing and linearly independent. If this occurs, the consistency condition (C3) implies that the product of the links along any path has $N_{c}$ unit eigenvalues. As the product is an $\mathrm{SU}\left(N_{c}\right)$ matrix, it must be equal to the identity matrix, which implies that all gauge fields are equivalent to the identity modulo gauge transformations. This argument shows therefore that the $\mathrm{U}(1)$ symmetry can be broken only if the relevant configurations are characterized by the triviality of the gauge fields. For $N_{c} \geq 3$ and $\beta_{g}=0$, this does not occur and the $\mathrm{U}(1)$ symmetry is unbroken. For $\beta_{g}=\infty$, there is no gauge dependence and the $\mathrm{U}(1)$ symmetry is broken, an obvious result given that the $\mathrm{U}(1)$ group is a subgroup of the larger $\mathrm{O}\left(2 N_{f} N_{c}\right)$ group. As we expect the gauge energy $E_{g}$ to depend smoothly on $\beta_{g}$, we should always have $E_{g}<1$ for finite $\beta_{g}$ : there are relevant nontrivial gauge configurations that always forbid the breaking of the U(1) symmetry.

Let us finally discuss the behavior for $N_{c}=2$. In this case, we can set $U_{\boldsymbol{x}, \mu}=1$ everywhere. Eq. (C1) implies that $Z_{\boldsymbol{x}}^{a f}$ takes the same value on each link. Thus, the average of any quantity $\mathcal{O}\left(Z_{\boldsymbol{x}}\right)$ can be obtained as

$$
\left\langle\mathcal{O}\left(\left(Z_{\boldsymbol{x}}\right)\right\rangle=\int[d A] \mathcal{O}(A),\right.
$$

where $A$ is an $N_{c} \times N_{f}$ matrix $\left(N_{c}=2\right)$ and $[d A]$ is the normalized invariant integration measure over the $N_{c} N_{f^{-}}$ dimensional complex sphere defined by $\operatorname{Tr} A^{\dagger} A=1$.

We obtain

$$
\left\langle\operatorname{Tr} P_{\boldsymbol{x}}^{2}\right\rangle=\int[d A] \operatorname{Tr}\left[\left(A^{\dagger} A\right)^{2}\right]=\frac{N_{f}+N_{c}}{1+N_{f} N_{c}},
$$

and

$$
U=\frac{\left(1+N_{f} N_{c}\right)\left(N_{f} N_{c}+4 N_{f}^{2}+N_{f}^{3} N_{c}-6\right)}{\left(N_{f}^{2}-1\right)\left(2+N_{f} N_{c}\right)\left(3+N_{f} N_{c}\right)}
$$

which again are consistent with the numerical data in Tables III and IV for $N_{c}=2$. Note that the results for $U$ are consistent with $U_{\Gamma}=1$ when $N_{f}=2$, see Eq. (A15).

The results that we have obtained for $N_{c}=2$ do not depend on the dimensionality of the system. On the other hand, for $N_{c} \geq 3$ the conclusions we have obtained rely on the fact that the relevant configurations have the form (C5) and (C6), a claim that is only justified by the numerical study we have performed on cubic lattices. We expect, but we do not have a proof, that the same result holds in any dimension.
[1] P. W. Anderson, Basic Notions of Condensed Matter Physics, (The Benjamin/Cummings Publishing Company, Menlo Park, California, 1984).

[2] S. Weinberg, The Quantum Theory of Fields, (Cambridge University Press, Cambridge, England, 2005).

[3] P. W. Anderson, Plasmons, Gauge Invariance, and Mass, Phys. Rev. 130, 439 (1963); Superconductivity: Higgs, Anderson and all that, Nat. Phys. 11, 93 (2015).

[4] S. Sachdev, Topological order, emergent gauge fields, and Fermi surface reconstruction, Rep. Prog. Phys. 82, 014001 (2019).

[5] L. D. Landau and E. M. Lifshitz, Statistical Physics. Part $I$, 3rd edition (Elsevier Butterworth-Heinemann, Oxford, 1980).

[6] K. G. Wilson and J. Kogut, The renormalization group and the $\epsilon$ expansion, Phys. Rep. 12, 75 (1974).

[7] M. E. Fisher, The renormalization group in the theory of critical behavior, Rev. Mod. Phys. 47, 543 (1975).

[8] A. Pelissetto and E. Vicari, Critical Phenomena and Renormalization Group Theory, Phys. Rep. 368, 549 (2002).

[9] J. Zinn Justin Quantum Field Theory and Critical Phenomena, (Oxford University Press, Oxford, 2002).

[10] T. Senthil, L. Balents, S. Sachdev, A. Vishwanath, and M. P. A. Fisher, Quantum criticality beyond the LandauGinzburg-Wilson paradigm, Phys. Rev. B 70, 144407 (2004).

[11] C. Wang, A. Nahum, M. A. Metlitski, C. Xu, and T. Senthil, Deconfined Quantum Critical Points: Symmetries and Dualities, Phys. Rev. X 7, 031051 (2017).

[12] R. D. Pisarski and F. Wilczek, Remarks on the chiral phase transition in chromodynamics, Phys. Rev. D 29, 338 (1984).

[13] A. Butti, A. Pelissetto, and E. Vicari, On the nature of the finite-temperature transition in QCD, J. High Energy Phys. 08, 029 (2003).

[14] A. Pelissetto and E. Vicari, Relevance of the axial anomaly at the finite-temperature chiral transition in QCD, Phys. Rev. D 88, 105018 (2013).

[15] M. D'Elia, High-Temperature QCD: theory overview, Nucl. Phys. A 982, 99 (2019).

[16] S. Sharma, Recent Progress on the QCD Phase Diagram, PoS LATTICE 2018, 009 (2019).

[17] Y.-Z. You, Y.-C. He, C. Xu, and A. Vishwanath, Symmetric Fermion Mass Generation as Deconfined Quantum Criticality, Phys. Rev. X 8, 011026 (2018).

[18] Y.-Z. You, Y.-C. He, A. Vishwanath, and C. Xu, From bosonic topological transition to symmetric fermion mass generation, Phys. Rev. B 97, 125112 (2018).

[19] A. Thomson and S. Sachdev, Fermionic Spinon Theory of Square Lattice Spin Liquids near the Néel State, Phys. Rev. X 8, 011012 (2018).

[20] A. Pelissetto and E. Vicari, Three-dimensional ferromagnetic CP ${ }^{N-1}$ models, Phys. Rev. E 100, 022122 (2019).

[21] A. Pelissetto and E. Vicari, Multicomponent compact Abelian-Higgs lattice models, Phys. Rev. E 100, 042134 (2019).

[22] A. Nahum, J. T. Chalker, P. Serna, M. Ortuño, and A. M. Somoza, 3D loop models and the $\mathrm{CP}^{n-1}$ sigma model, Phys. Rev. Lett. 107, 110601 (2011).

[23] A. Nahum, J. T. Chalker, P. Serna, M. Ortuño, and A. M. Somoza, Phase transitions in three-dimensional 


\begin{tabular}{|c|c|c|c|c|c|c|}
\hline$\overline{\left(N_{c}, N_{f}\right)}$ & $\overline{E g}$ & $E / 3$ & $\bar{U}$ & Eq. (C11) & $\operatorname{Tr} P^{2}$ & Eq. (C10) \\
\hline$(2,2)$ & $0.99998(2)$ & $-0.999992(6)$ & $1.191(2)$ & 1.19048. & $0.800(1)$ & 0.8 \\
\hline$(2,3)$ & $1.00000(1)$ & $-1.000010(5)$ & $1.0940(5)$ & $1.09375 \ldots$ & $0.714(2)$ & 0.714286 . \\
\hline$(2,4)$ & $1.00001(1)$ & $-1.000000(4)$ & $1.0582(3)$ & 1.05818. & $0.666(1)$ & 0.666667 . \\
\hline$(2,5)$ & $1.00000(1)$ & $-1.000000(6)$ & $1.0400(3)$ & 1.04006. & $0.636(1)$ & $0.636364 \ldots$ \\
\hline$(2,6)$ & $0.999990(6)$ & $-1.000000(4)$ & $1.0294(2)$ & 1.02939. & $0.615(2)$ & $0.615385 \ldots$ \\
\hline$(3,2)$ & $0.3347(3)$ & $-1.000005(6)$ & $1.0000000(1)$ & & $1.00001(1)$ & \\
\hline$(3,3)$ & $0.3369(4)$ & $-0.999993(6)$ & $1.0000000(6)$ & & $0.99999(1)$ & \\
\hline$(3,4)$ & $0.3382(4)$ & $-1.000000(7)$ & $1.0000000(6)$ & & $1.00000(1)$ & \\
\hline$(3,5)$ & $0.3413(4)$ & $-1.000000(5)$ & $1.0000000(1)$ & & $1.00000(1)$ & \\
\hline$(3,6)$ & $0.3463(4)$ & $-1.000000(5)$ & $1.00000000(4)$ & & $0.99999(1)$ & \\
\hline$(4,2)$ & $0.2500(2)$ & $-0.99999(1)$ & $1.0000000(4)$ & & $1.00000(1)$ & \\
\hline$(4,3)$ & $0.2510(2)$ & $-1.00001(1)$ & $1.0000000(2)$ & & $1.00001(2)$ & \\
\hline$(4,4)$ & $0.2513(3)$ & $-0.99995(4)$ & $1.0000000(3)$ & & $0.99992(8)$ & \\
\hline$(4,5)$ & $0.2520(4)$ & $-1.000010(7)$ & $1.0000000(1)$ & & $1.00002(2)$ & \\
\hline$(4,6)$ & $0.2527(3)$ & $-1.000000(6)$ & $1.0000000(1)$ & & $0.99999(1)$ & \\
\hline
\end{tabular}

TABLE III: Asymptotic values for $\beta \rightarrow \infty$ on a $2^{3}$ lattice at $\beta_{g}=0$.

\begin{tabular}{|c|c|c|c|c|c|c|}
\hline$\left(N_{c}, N_{f}\right)$ & $E_{g}$ & $E / 3$ & $U$ & Eq. (C11) & $\operatorname{Tr} P^{2}$ & Eq. C10 \\
\hline$(2,2)$ & $0.99998(2)$ & $-0.999999(2)$ & $1.195(3)$ & 1.19048. & $0.798(1)$ & 0.8 \\
\hline$(2,3)$ & $1.000000(6)$ & $-1.000000(2)$ & $1.094(1)$ & 1.09375 . & $0.714(3)$ & 0.714286 . \\
\hline$(2,4)$ & $0.999999(3)$ & $-1.000000(3)$ & $1.0580(5)$ & 1.05818 . & $0.666(2)$ & 0.666667 . \\
\hline$(2,5)$ & $1.000000(3)$ & $-1.000000(3)$ & $1.040(1)$ & 1.04006. & $0.637(2)$ & 0.636364 . \\
\hline$(2,6)$ & $1.000000(3)$ & $-0.999988(5)$ & $1.0293(5)$ & 1.02939. & $0.615(2)$ & 0.615385. \\
\hline$(3,2)$ & $0.3344(2)$ & $-1.000000(3)$ & $1.000000(3)$ & & $1.000000(6)$ & \\
\hline$(3,3)$ & $0.3356(2)$ & $-1.000000(3)$ & $1.000000(3)$ & & $0.999999(6)$ & \\
\hline$(3,4)$ & $0.3368(2)$ & $-0.999999(3)$ & $1.000000(3)$ & & $1.000008(8)$ & \\
\hline$(3,5)$ & $0.3375(2)$ & $-1.000000(3)$ & $1.00000000(5)$ & & $1.00001(1)$ & \\
\hline$(3,6)$ & $0.3385(2)$ & $-0.999996(4)$ & $1.00000000(2)$ & & $0.99999(1)$ & \\
\hline$(4,2)$ & $0.2501(2)$ & $-1.00000(1)$ & $1.0000000(1)$ & & $0.99999(2)$ & \\
\hline
\end{tabular}

TABLE IV: Asymptotic values for $\beta \rightarrow \infty$ on a $4^{3}$ lattice at $\beta_{g}=0$.

loop models and the $C P^{n-1}$ sigma model, Phys. Rev. B 88, 134411 (2013).

[24] A. Pelissetto, A. Tripodo, and E. Vicari, LandauGinzburg-Wilson approach to critical phenomena in the presence of gauge symmetries, Phys. Rev. D 96, 034505 (2017).

[25] A. Pelissetto, A. Tripodo and E. Vicari, Criticality of $\mathrm{O}(\mathrm{N})$ symmetric models in the presence of discrete gauge symmetries, Phys. Rev. E 97, 012123 (2018).

[26] S. Gazit, F. F. Assaad, S. Sachdev, A. Vishwanath, and C. Wang, Confinement transition of $Z_{2}$ gauge theories coupled to massless fermions: emergent $\mathrm{QCD}_{3}$ and $\mathrm{SO}(5)$ symmetry, Proc. Natl. Acad. Sci. 115, E6987 (2018).

[27] S. Sachdev, H. D. Scammell, M. S. Scheurer, and G. Tarnopolsky, Gauge theory for the cuprates near optimal doping, Phys. Rev. B 99, 054516 (2019).

[28] S. Nadkarni, The SU(2) Adjoint Higgs Model in Three dimensions, Nucl. Phys. B 334, 559 (1990).

[29] K. Kajantie, K. Rummukainen and M. E. Shaposhnikov, A Lattice Monte Carlo study of the hot electroweak phase transition, Nucl. Phys. B 407, 356 (1993).
[30] W. Buchmüller and O. Philipsen, Phase structure and phase transition of the $\mathrm{SU}(2)$ Higgs model in threedimensions, Nucl. Phys. B 443, 47 (1995).

[31] K. Kajantie, M. Laine, K. Rummukainen, and M. E. Shaposhnikov, Is there a hot electroweak phase transition at $m_{H} \gtrsim m_{W}$ ?, Phys. Rev. Lett. 77, 2887 (1996).

[32] A. Hart, O. Philipsen, J. D. Stack, and M. Teper, On the phase diagram of the $\mathrm{SU}(2)$ adjoint Higgs model in (2+1)-dimensions, Phys. Lett. B 396, 217 (1997).

[33] K. Osterwalder and E. Seiler, Gauge Field Theories on the Lattice, Ann. Phys. (NY) 110, 440 (1978).

[34] E. Fradkin and S. Shenker, Phase diagrams of lattice gauge theories with Higgs fields, Phys. Rev. D 19, 3682 (1979).

[35] S. Dimopoulos, S. Raby, and L. Susskind, Light Composite Fermions, Nucl. Phys. B 173, 208 (1980).

[36] C. Bonati, A. Pelissetto, and E. Vicari, Phase diagram, symmetry breaking, and critical behavior of threedimensional lattice multiflavor scalar chromodynamics, Phys. Rev. Lett. 123, 232002 (2019).

[37] K. G. Wilson, Confinement of quarks, Phys. Rev. D 10, 
2445 (1974).

[38] H. Georgi, Weak interactions and modern particle theory, (The Benjamin/Cummings Publishing Company, Menlo Park, California, 1984).

[39] P. Arnold and L. G. Yaffe, The $\epsilon$ expansion and the electroweak phase transition, Phys. Rev. D 49, 3003 (1994).

[40] P. S. Bhupal Dev and A. Pilaftsis, Maximally Symmetric Two Higgs Doublet Model with Natural Standard Model Alignment, JHEP 1412, 024 (2014); (Erratum) JHEP 1511, 147 (2015).

[41] B. Simon Representations of Finite and Compact Groups (American Mathematical Society, Providence, 1996).

[42] A. Das, Phase transition in $\mathrm{SU}(N) \times \mathrm{U}(1)$ gauge theory with many fundamental bosons, Phys. Rev. B 97, 214429 (2018).

[43] V. Alba, A. Pelissetto, and E. Vicari, The Uniformly Frustrated Two-Dimensional $X Y$ Model in the Limit of Weak Frustration, J. Phys. A: Math. Theor. 41, 175001 (2008).

[44] M. Creutz, Monte Carlo Study of Quantized SU(2) Gauge Theory, Phys. Rev. D 21, 2308 (1980).

[45] A. D. Kennedy and B. J. Pendleton, Improved Heat Bath Method for Monte Carlo Calculations in Lattice Gauge Theories, Phys. Lett. 156B, 393 (1985).

[46] M. Creutz, Overrelaxation and Monte Carlo Simulation, Phys. Rev. D 36, 515 (1987).

[47] N. Cabibbo and E. Marinari, A New Method for Updating $\mathrm{SU}(\mathrm{N})$ Matrices in Computer Simulations of Gauge Theories, Phys. Lett. 119B, 387 (1982).

[48] N. Metropolis, A. W. Rosenbluth, M. N. Rosenbluth, A. H. Teller and E. Teller, Equation of state calculations by fast computing machines, J. Chem. Phys. 21, 1087 (1953).

[49] S. A. Antonenko and A. I. Sokolov, Critical exponents for a three-dimensional $\mathrm{O}(n)$-symmetric model with $n>3$,
Phys. Rev. E 51, 1894 (1995).

[50] M. Hasenbusch, A. Pelissetto, and E. Vicari, Instability of the $\mathrm{O}(5)$ critical behavior in the $\mathrm{SO}(5)$ theory of high$T_{c}$ superconductors, Phys. Rev. B 72014532 (2005).

[51] L. A. Fernández, V. Martín-Mayor, D. Sciretti, A. Tarancón, and J. L. Velasco, Numerical study of the enlarged $\mathrm{O}(5)$ symmetry of the 3-D antiferromagnetic $\mathrm{RP}^{2}$ spin model, Phys. Lett. B 628, 281 (2005).

[52] P. Calabrese, A. Pelissetto, and E. Vicari, Multicritical behavior of $\mathrm{O}\left(n_{1}\right) \oplus \mathrm{O}\left(n_{2}\right)$-symmetric systems, Phys. Rev. B 67, 054505 (2003).

[53] F. Delfino, A. Pelissetto, and E. Vicari, Threedimensional antiferromagnetic $\mathrm{CP}^{N-1}$ models, Phys. Rev. E 91, 052109 (2015).

[54] M. Hasenbusch and E. Vicari, Anisotropic perturbations in 3D $\mathrm{O}(N)$ vector models, Phys. Rev. B 84, 125136 (2011).

[55] M. Campostrini, M. Hasenbusch, A. Pelissetto, P. Rossi, and E. Vicari, Critical exponents and equation of state of the three-dimensional Heisenberg universality class, Phys. Rev. B 65, 144520 (2002).

[56] R. Guida and J. Zinn-Justin, Critical exponents of $N$ vector models, J. Phys. A 31, 8103 (1998).

[57] M. Campostrini, A. Pelissetto, P. Rossi, and E. Vicari, Four-point renormalized coupling in $\mathrm{O}(N)$ models, Nucl. Phys. B 459, 207 (1996).

[58] M. S. S. Challa, D. P. Landau, and K. Binder, Finitesize effects at temperature-driven first-order transitions Phys. Rev. B 34, 1841 (1986).

[59] K. Vollmayr, J. D. Reger, M. Scheucher, and K. Binder, Finite size effects at thermally-driven first order phase transitions: A phenomenological theory of the order parameter distribution Z. Phys. B 91113 (1993). 